\title{
Differentiated Participation of Thalamocortical Subnetworks in Slow/Spindle Waves and Desynchronization
}

\author{
Mika Ushimaru, ${ }^{1,2,3}$ Yoshifumi Ueta, ${ }^{1,3}$ and Yasuo Kawaguchi ${ }^{1,2,3}$ \\ ${ }^{1}$ Division of Cerebral Circuitry, National Institute for Physiological Sciences, and ${ }^{2}$ Department of Physiological Sciences, Graduate University for Advanced \\ Studies (Sokendai), Okazaki 444-8787, Japan, and 3Japan Science and Technology Agency, CREST, Tokyo 102-0076, Japan
}

During sleep, the electroencephalogram exhibits synchronized slow waves that desynchronize when animals awaken [desynchronized states (DSs)]. During slow-wave states, the membrane potentials of cortical neurons oscillate between discrete depolarized states ("Up states") and periods of hyperpolarization ("Down states"). To determine the role of corticothalamic loops in generating Up/Down oscillations in rats, we recorded unit activities of layer 5 (L5) corticothalamic (CTh) cells in the frontal cortex, neurons in the thalamic reticular nucleus, and basal ganglia- and cerebellum-linked thalamic relay nuclei, while simultaneously monitoring the local cortical field potential to identify slow-wave/spindle oscillations and desynchronization. We found that (1) some basal ganglia-linked and reticular thalamic cells fire preferentially near the beginning of Up states; (2) thalamic cells fire more selectively at a given Up-state phase than do CTh cells; (3) CTh and thalamic cells exhibit different action potential timings within spindle cycles; and (4) neurons exhibit different firing characteristics when comparing their activity during Up states and DSs. These data demonstrate that cortico-thalamo-cortical subnetworks are temporally differentiated during slow and spindle oscillations, that the basal ganglia-linked thalamic nuclei are closely related with Up-state initiation, and that Up states and DSs are distinguished as different depolarization states of neurons within the network.

\section{Introduction}

Activity within the neocortex is very different during sleep and wakefulness. The electroencephalogram (EEG) during deep sleep is characterized by $<1 \mathrm{~Hz}$ slow waves, which desynchronize when animals are waking (Steriade et al., 2001). During slow-wave states (SWSs), membrane potentials of cortical neurons fluctuate between discrete depolarizing events, called "Up states," and inter-Up-state hyperpolarizations, known as "Down states" (Metherate and Ashe, 1993; Steriade et al., 1993; Cowan and Wilson, 1994). Transitions between Up and Down states are well synchronized across cortical areas (Amzica and Steriade, 1995). Up-state depolarizations are assumed to start from L5 (Sanchez-Vives and McCormick, 2000), but thalamic inputs may play an important role for the initiation of cortical Up states (Rigas and CastroAlamancos, 2007; Crunelli and Hughes, 2010).

Desynchronized states (DSs), sustained depolarized states, are considered to be essential for receiving inputs from the periphery and other brain regions, whereas the functional significance of Up states has received relatively little attention (Destexhe et al., 2007). Up states have been hypothesized to be fragments of the

Received Sept. 25, 2011; accepted Nov. 30, 2011.

Author contributions: M.U. and Y.K. designed research; M.U. and Y.U. performed research; M.U. and Y.K. analyzed data; M.U. and Y.K. wrote the paper.

This work was supported by Japan Science and Technology Agency, CREST, and by Grant-in-Aids for Scientific Research from the Ministry of Education, Culture, Sports, Science, and Technology. We thank Dr. M. Victoria Puig for helpful advice, Mika Watanabe for histological assistance, and Drs. Allan T. Gulledge and Fuyuki Karube for comments on this manuscript.

Correspondence should be addressed to Yasuo Kawaguchi, Division of Cerebral Circuitry, National Institute for Physiological Sciences, Okazaki 444-8787, Japan. E-mail:yasuo@nips.ac.jp.

DOI:10.1523/JNEUROSCI.4883-11.2012

Copyright $\odot 2012$ the authors $\quad 0270-6474 / 12 / 321730-17 \$ 15.00 / 0$
DSs, since both are depolarizations across broad populations of neurons (Destexhe et al., 2007).

Up-state-like depolarization at the slow-wave frequency, induced by electrical stimulation outside the scalp during sleep, promotes consolidation of declarative memory (Marshall et al., 2006). Patterns of action potential generation in cortical populations during sleep are not random but reflect those observed during wakefulness (Louie and Wilson, 2001; Euston et al., 2007). These data suggest that some information processing occurs in local circuits during Up-state depolarizations (Buzsáki, 1998). To coordinate specific firing sequences among cortical neurons during SWS, faster oscillations may be required. Indeed, spindle waves $(7-14 \mathrm{~Hz}$ ) are nested within Up states (Steriade, 2006), and their rhythm is generated in the thalamic reticular nucleus (Steriade et al., 1987).

To better understand Up-state initiation, firing phase differentiation during Up/Down states and spindle cycles, and differences in cortico-thalamo-cortical communication during Up states and DS, we compared the firing patterns of L5 corticothalamic (CTh) cells in the frontal cortex with thalamic cells in ventrolateral (VL), ventral anterior/ventromedial (VA/VM), and reticular (Rt) nuclei. These four neuron subtypes were chosen for the following reasons: (1) activity of L5 CTh cells generate higher amplitude EPSPs in the thalamus than do layer 6 CTh cells, and are therefore considered to provide "driver" input to the thalamus (Sherman and Guillery, 1998). Furthermore, Up states commence in L5, and L5 CTh cells are suspected of playing an important role in corticothalamic rhythm generation and synchronization. (2) In the frontal cortex, it is known that thalamocortical inputs come from two major groups of thalamic nuclei: 
VL and VA/VM nuclei (Jones, 2001, 2007). The former receives input from cerebellum and innervates mainly the middle cortical layers, whereas the latter relays information from basal ganglia and innervates layer 1. (3) Neurons in the Rt nucleus provides inhibition to thalamic relay cells and participate in spindle generation (Pinault, 2004).

By comparing unit activities in these four distinct subtypes, we identified cell type-specific temporal firing patterns during Up/ Down states and spindle cycles, as well as distinct firing patterns that differ between Up states and DSs.

\section{Materials and Methods}

Anterograde and retrograde tracer injections. The present experiments were approved by the Institutional Animal Care and Use Committee of National Institutes of Natural Sciences. Male Wistar rats (4 weeks of age) were anesthetized with a mixture of ketamine $(40 \mathrm{mg} / \mathrm{kg}$, i.p.) and xylazine $(4 \mathrm{mg} / \mathrm{kg}$, i.p.) and placed in a stereotaxic apparatus. A small area of the skull overlying dorsal part of frontal cortex including the secondary motor area (M2) was removed. Glass pipettes were filled with $0.5 \mathrm{M}$ potassium acetate including $5 \%(\mathrm{w} / \mathrm{v})$ biotinylated dextran amine (BDA) (BDA-10000; Invitrogen) and 0.2\% (w/v) Alexa Fluor 555-conjugated cholera toxin subunit b (CTB555) (Invitrogen), and advanced into the cortex with rostral inclination of $25^{\circ}$ from the vertical. The tracers (total injected solution, $100 \mathrm{nl})$ were deposited within M2 $(4.0$ or $4.5 \mathrm{~mm}$ anterior to the bregma; eight divided injections: 1.3 and $1.7 \mathrm{~mm}$ lateral to the midline, each with depths of $0.2,0.4,0.6$, and $0.8 \mathrm{~mm}$ from the surface) by pressure application (PV820; WPI) (see Figs. 2, 3A). After a survival period of $7 \mathrm{~d}$, the animals were deeply anesthetized with sodium pentobarbital (60 mg/kg, i.p.), and then perfused transcardially with 10 $\mathrm{ml}$ of a prefixative solution of $250 \mathrm{~mm}$ sucrose, $5 \mathrm{~mm} \mathrm{MgCl}_{2}$ in $0.02 \mathrm{M}$ phosphate buffer, $\mathrm{pH} 7.4(\mathrm{~PB})$, followed by a fixative solution containing $4 \%$ paraformaldehyde and $0.2 \%$ picric acid in $0.1 \mathrm{M} \mathrm{PB}$. The brain was sagittally cut into $50 \mu \mathrm{m}$ sections on a vibratome (Leica). The BDA was revealed by incubating sections in a 1:100 dilution of avidin-biotinylated horseradish peroxidase complex (ABC Elite; Vector Laboratories) in Tris-HCl-buffered saline (TBS) $(0.05 \mathrm{M})$ overnight. To enhance the BDA signal, sections were reacted for $30 \mathrm{~min}$ with $2.5 \mu \mathrm{M}$ biotinylated tyramine, $3 \mu \mathrm{g} / \mathrm{ml}$ glucose oxidase, and $2 \mathrm{mg} / \mathrm{ml} \beta$-D-glucose. Sections were subsequently incubated with Alexa Fluor-conjugated streptavidin (Invitrogen). Thalamic nuclei and subregions were identified by simultaneous immunostaining for calbindin and NeuN (see below).

Electrophysiology: single-unit, local field potential recordings and electrical stimulation. Male Wistar rats ( 5 weeks of age) were used. The animals were anesthetized with chloral hydrate (initial dose, $400 \mathrm{mg} / \mathrm{kg}$, i.p.; supplemental doses, $80 \mathrm{mg} / \mathrm{kg}$, i.v., through the femoral vein every $\sim 45 \mathrm{~min}$ ) and placed in a stereotaxic frame. Body temperature was maintained at $37^{\circ} \mathrm{C}$ throughout the experiment with a heating pad. Lidocaine was used at all wound margins and points of contact between the animal and the stereotaxic equipment to reduce any incidental pain. The intracortical local field potential (LFP) and animal movement were monitored carefully. At the end of each cycle of anesthesia, we recorded an extra 10-20 $s$ during which the animals did not show any sign of consciousness, despite having desynchronized LFPs (DS, indicated by a total cessation of slow waves and the absence of spindles). Immediately after desynchronization, animals were given a supplemental dose of anesthetic that generated slow oscillations within several tens of seconds.

Single-unit (sampling rate, $16 \mathrm{kHz}$ ) and LFP (sampling rate, $1200 \mathrm{~Hz}$ ) activities were recorded with Axoclamp $2 \mathrm{~B}$ amplifiers and Spike2 software (Cambridge Electronic Design) in M2 (rostral medial agranular cortex; AP, 2.7-3.7; L, 1.3-1.9; DV, 0.5-2 mm) and thalamus (AP, -1.5 to -4 ; L, 1.3-1.9; DV, $4-8 \mathrm{~mm}$ ). Recordings were made with glass pipettes (15-50 M $\Omega$ ) filled with $0.5 \mathrm{M} \mathrm{NaCl}$ containing $2 \%$ Neurobiotin (w/v; Vector Laboratories). Unit and LFP signals were filtered on-line at $0.3-3 \mathrm{kHz}$ and $0.1-200 \mathrm{~Hz}$, respectively. The additional LFP activity was recorded in L5 of M2 from another glass electrode filled with $2 \mathrm{M} \mathrm{NaCl}$ (15-25 M $\Omega$ ).

In the case of CTh cell recording, bipolar stimulating electrodes were stereotaxically implanted in the thalamus (AP, $-3 ; \mathrm{L}, 1.5 ; \mathrm{DV}, 7 \mathrm{~mm})$ in each animal. Stimulating electrodes consisted of two stainless-steel enamel-coated wires (California Fine Wire; size, 0.005 BF; stainless steel 304, H-ML, H-ML/ML bifilar). Stimulations were monophasic square wave pulses of $0.2 \mathrm{~ms}$ at $1 \mathrm{~Hz}(0.1-1 \mathrm{~mA})$.

Neuron labeling and histology. At the end of the recording session, the neurons were stained by juxtacellular injection of Neurobiotin using 600 $\mathrm{ms}$ cycles of positive current pulses ( $250 \mathrm{~ms}$ on/350 ms off, $1-8 \mathrm{nA}, 5-20$ min) (Pinault, 1996). In addition, recorded site of LFP was marked with $2 \%(\mathrm{w} / \mathrm{v})$ Neurobiotin in $0.5 \mathrm{M} \mathrm{NaCl}$ ( $7 \mathrm{~s}$ on/off, $10 \mu \mathrm{A}, 1-2 \mathrm{~min}$ ) or by current injection ( $7 \mathrm{~s}$ on/off, $10 \mu \mathrm{A}, 1-2 \mathrm{~min}$ ). In the case of paired recording of LFP, the other recording site was marked with 7\% (w/v) Fast Green (Fast Green FCF; Sigma-Aldrich) in $2 \mathrm{M} \mathrm{NaCl}$ by anodal current ( 7 s on/off, 8-10 $\mu \mathrm{A}, 8-10 \mathrm{~min}$ ).

Two to $4 \mathrm{~h}$ after injection, rats were perfused through the heart with the prefixative solution, followed by $300 \mathrm{ml}$ of fixative containing $4 \%$ paraformaldehyde, $0.05 \%$ glutaraldehyde, and $0.2 \%$ picric acid in $0.1 \mathrm{M}$ $\mathrm{PB}$. After postfixation, the brain was removed and kept in fixative overnight. The brain was then sliced, and the positions of the stimulating electrodes were confirmed.

For visualization of Neurobiotin-injected cells, the brain was sliced at a thickness of $50 \mu \mathrm{m}$. Sections were incubated with avidin-biotinylated horseradish peroxidase complex (1:100; Vector Laboratories) in $0.05 \mathrm{M}$ TBS with $0.1-0.5 \%$ Triton X-100 (TX) overnight at $4^{\circ} \mathrm{C}$. After washing in TBS, the slices were reacted with 3,3'-diaminobenzidine tetrahydrochloride (DAB) $(0.02 \%)$, nickel ammonium sulfate $(0.3 \%)$, and $\mathrm{H}_{2} \mathrm{O}_{2}$ $(0.01 \%)$ in Tris- $\mathrm{HCl}$ buffer. They were then postfixed in $1 \% \mathrm{OsO}_{4}$ in 0.1 M PB containing $7 \%$ glucose, dehydrated, and flat-embedded on glass slides in Epon (see Fig. 4C,D).

Identification of thalamic nuclei and frontal cortical layers. Thalamic nuclei and subregions were identified by simultaneous immunostaining for calbindin (rabbit antiserum; Swant; CB-38a; 1:2000) and NeuN (mouse monoclonal antibody; Millipore Bioscience Research Reagents; MAB377; 1:1000). After visualization of recorded thalamic cell with DAB reaction, the section containing the soma was washed in TBS and then incubated with rabbit calbindin and mouse NeuN antibodies in TBS containing $10 \%$ normal goat serum, $2 \%$ bovine serum albumin, and $0.1 \%$ TX overnight. After washes with TBS, sections were incubated with a mixture of Alexa 488- and Alexa 594-conjugated secondary antibodies (see Fig. $4 E, F$ ).

Frontal cortical layers were cytologically identified on the basis of the size and density of neuronal somata: layer 1 (L1) is the most superficial cell-sparse layer; layer 5 (L5) contains cells with larger somata than superficial layers and layer 6 (L6); and L6 is higher in density of somata than L5. L1 and layer 2/3 (L2/3), a layer between L1 and L5, could be further divided into sublayers different in vesicular glutamate transporter type 2 (VGLUT2) immunoreactivity (Morishima et al., 2011): upper L1 (L1a) with stronger VGLUT2 immunoreactivity than lower L1 (L1b); and lower L2/3 (L2/3b) with stronger immunoreactivity than upper layer 2/3 (L2/3a), indicating the major thalamic recipient zones. For sublayer identification, sections were incubated with a guinea pig antibody against VGLUT2 (1:2000; AB2251; Millipore Bioscience Research Reagents) in TBS containing $10 \%$ normal goat serum, $2 \%$ bovine serum albumin, and $0.1 \%$ TX in TBS overnight at $4^{\circ} \mathrm{C}$. Sections were then incubated with an Alexa 488-conjugated secondary antibody.

Data analysis. To quantify the burst firing strength, we calculated the burst index as follows: (number of spike interval $<10 \mathrm{~ms}$ )/(number of spike interval $<200 \mathrm{~ms}$ ) (see Fig. $5 D$ ).

The beginning and end of Up states were determined by measuring changes in the $20-100 \mathrm{~Hz}$ components of the LFP, following a method proposed by Mukovski et al. (2007). Briefly, 20-100 Hz component of the cortical LFP was extracted and durations with high SD in this band width were defined as Up states. If two Up states or two Down states were separated by $<40 \mathrm{~ms}$, the two states were extended to fill the gap and pasted together (Volgushev et al., 2006). Up/Down-state border crossings for periods $<40 \mathrm{~ms}$ were not considered as state shifts. To examine the firing strength around the Up-state onset, we calculated two parameters: (1) firing frequency around Up starts in $100 \mathrm{~ms}$ bin and (2) Upstart spike number ratio [(number of spikes $-100 \mathrm{~ms}<$ Up onset $<100$ $\mathrm{ms}) /($ total spikes during SWS)] (see Fig. 6). 

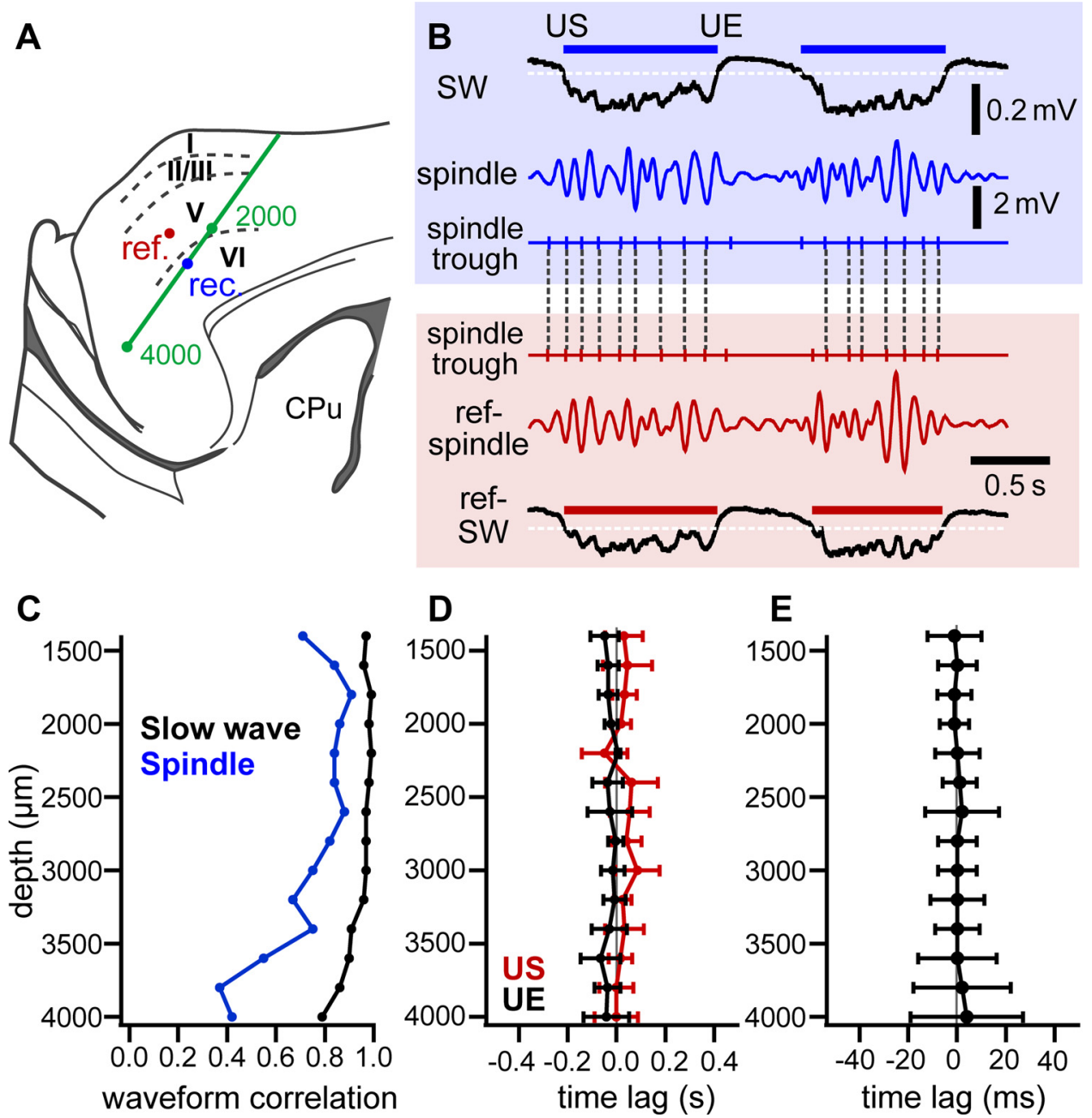

Figure 1. Up/Down state and spindle wave synchronization within the frontal cortex. $A$, An electrode track (green) for recording LFP at various locations in M2, and recorded point of the reference LFP (ref) (red circle). $\boldsymbol{B}$, Slow and spindle wave recordings at one depth (blue top; $2600 \mu \mathrm{m} ; \boldsymbol{A}$, blue circle) and the reference (red bottom; $\boldsymbol{A}$, red circle). SW, Raw LFP showing slow waves. Simultaneously detected spindle troughs at the two points are connected by dotted lines. C, Depth profile of slow (black) and spindle (blue) waveform correlations. D, Relationship of Up-state start (US) and end (UE) in individual depths to the reference point, expressed as time difference. Seven and 1 of 92 recorded pairs were significantly different in US and UE time difference, respectively $(p<0.05$; post hoc Tukey's test).E, Spindle trough relationship of individual depths to the reference point, expressed as time difference. No difference in spindle time was detected ( $p$ ost hoc Tukey's test).

To quantify the firing timing in Up/Down states, we normalized each duration to 1 ( -0.5 to 0.5 ) and made spike histograms (see Figs. 7-9). To evaluate the specificity of firing times, the $\chi^{2}$ test for goodness of fit was used (see Figs. 7,8$). \chi^{2}$ value was calculated as $\Sigma[$ (observed - expected $)^{2}$ /expected], where observed was spike number in each bin, and expected was averaged spike number.

Spindle $(7-14 \mathrm{~Hz})$ bands were digitally filtered in Spike2 and big troughs that exceed fourfold of root mean square amplitude of those in Down-state duration were detected with PTLEV script (see Figs. 1, 10). Phase of detected troughs in L5 was defined as $0^{\circ}$. For each cell, we built phase histograms $\left(20^{\circ}\right.$ bin $)$ of spike timing relative to spindle troughs, and firing was considered to be modulated by the oscillation if $p<0.05$ using the Rayleigh tests (see Fig. 10). For this analysis, neurons firing at a low rate ( $<90$ spikes in phase histogram) were excluded. From the phase histograms, we computed the mean angle and the intensity of modulation $r$, which was the length of the mean vector of all phase angles normalized by the number of spikes/angle. To compare the modulated phase angle, a multisample Watson-Williams test was used. To detect the distribution uniformity in modulated angle in each neuron subtype, the Hodges-Ajne test for circular uniformity was used.

To quantify the firing frequency change from SWS to DS, DS preference of firing was defined as follows: (DSf - SWSf)/(DSf + SWSf), where DSf is the firing frequency in DS and SWSf in SWS (see Fig. 12).
Basic statistical analysis was performed by using Prism (GraphPad software). Circular statistics and LFP processing (to detect Up-state start/end) were computed in MATLAB (MathWorks). Data are expressed as mean \pm SD. Statistical significance was set at a 95\% confidence level (two-tailed). ANOVA was used for confirmation of significant differences among subtypes in individual parameters, followed by post hoc Tukey's test. For comparison of two cell classes, Mann-Whitney $U$ test and $\chi^{2}$ test were used.

\section{Results}

Wide-area synchronization in local field potential during SWS within M2

In SWS, Up/Down rhythms are repeated regularly around $1 \mathrm{~Hz}$ in LFP, but the duration of each epoch is not constant (Figs. $1 B$, $6 A)$. Up states contain nested higher frequency components including spindles $(7-14 \mathrm{~Hz}$ ) (Figs. $1 \mathrm{~B}, 10 \mathrm{~A}$ ). We confirmed that spindles, obtained by filtering of cortical LFP, were nested in Up states and disappeared in Down states (Fig. $1 B$ ). The troughs of spindle waves corresponded well to those on the Up states in the raw LFP (see Fig. 10Aa). To see how these rhythms are synchronized within cortical area M2, we investigated the temporal relationship of LFPs occurring at various points (200 

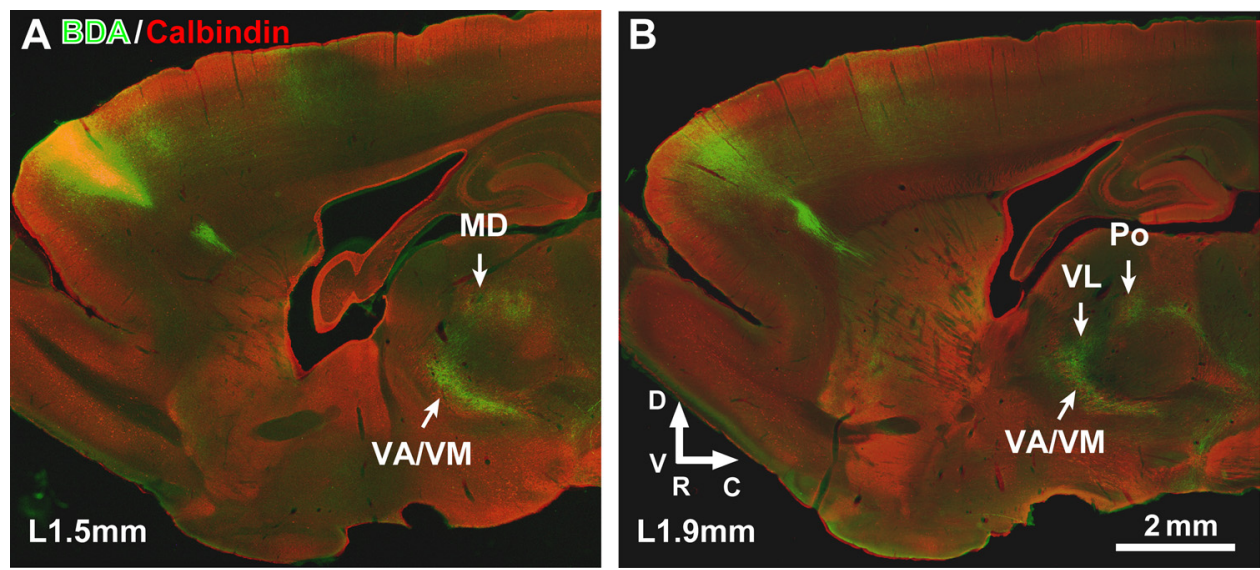

Figure 2. Corticothalamic projections from frontal cortex. Corticothalamic fibers were labeled with anterograde tracer (BDA; green fluorescence) injection into the secondary motor cortex. Red fluorescence, Calbindin immunoreactivity. $\boldsymbol{A}$, Sagittal section $1.5 \mathrm{~mm}$ lateral to midline. Note dense anterogradely labeled fibers in restricted parts of thalamus, including VA/VM and mediodorsal (MD) nuclei. $\boldsymbol{B}$, Sagittal section $1.9 \mathrm{~mm}$ lateral to midline. Note dense labeled fibers in restricted parts of thalamus, including VA/VM, VL, and posterior (Po) nuclei.

$\mu \mathrm{m}$ apart; 13-15 points in individual rats) across M2 cortex. For that purpose, we compared the slow/spindle waves and the start and end of Up states at individual points and one reference point (Fig. 1).

Both slow and spindle waves were well correlated (Fig. 1C). The waveform correlation was $0.94 \pm 0.02$ in slow wave, and $0.74 \pm 0.06$ in spindle wave (four rats). In comparison between any two points (Fig. $1 D, E$ ), only a few pairs were significantly different ( $p<0.05$; post hoc Tukey's test) in Up start (37 of 261 pairs in three rats), in Up end (34 of 261 pairs), and in spindle trough ( 1 of 261 pairs in three rats). These indicate that both spindle and slow waves are well synchronized within M2.

\section{Reciprocal connections between M2 and VL and VA/VM thalamic nuclei}

Cerebral cortex and thalamus are reciprocally connected and there is a possibility that interplay between these two regions is important for cortical synchronization. To confirm the reciprocal projections between thalamic nuclei and cortical area, we injected an anterograde (BDA) and a retrograde tracer (CTB555) simultaneously into M2 (Figs. 2, 3A). Anterogradely labeled fibers could be traced from M2 to VL and VA/VM nuclei in thalamus (Fig. 2). Anterogradely labeled terminals and retrogradely labeled somata were found in these thalamic nuclei. More importantly, the territories of anterogradely labeled terminals coincided with those of retrogradely labeled somata (Fig. 3C,E). We confirmed the same relationship of reciprocal connections in three rats.

\section{Recorded neuron type identification}

In the rat frontal agranular cortex, $\mathrm{L} 2 / 3$ can be divided into two sublayers (Morishima et al., 2011). L2/3b is dense in VGLUT2 immunoreactivity (Fig. 3A) and considered to be the thalamic inputrecipient zone, corresponding to layer 4 in sensory areas in aspect of extracortical inputs. We investigated the unit activity in this middle sublayer receiving thalamic inputs. L2/3b neurons were targeted for recordings, based on their cortical depth. However, post hoc laminar identification of recorded cells by juxtacellular staining and VGLUT2 immunohistochemistry showed that most of stained pyramidal cells were not inside L2/3b ( 11 of 12 cells: 4 cells at the border between L2/3a and L2/3b; 2 cells at the border between L2/3b and L5; 1 cell in L2/3a; and 4 cells in L5). Only one was found wholly within $\mathrm{L} 2 / 3 \mathrm{~b}$ (data not shown). These suggest that L2/3b pyramidal cells do not fire frequently during SWS.
Since L1 was also strongly innervated by thalamic fibers and L5 pyramidal cells develop rich dendritic tufts there, we next investigated L5 pyramidal cell activity during SWS. L5 of the rat frontal cortex is composed of several subtypes of projection cells (Morishima and Kawaguchi, 2006; Morishima et al., 2011; Otsuka and Kawaguchi, 2011). It would be important to investigate firing properties in identified pyramidal cell subtypes among spontaneously firing L5 neurons. Furthermore, it is well known that some subtypes of L 5 neurons project to the thalamus. Therefore, we targeted CTh neurons in L5 by identifying L5 neurons antidromically activated by electrical stimulation of the thalamus (around VL and VA/VM nuclei) (Fig. 4A). Based on the juxtacellular staining and recording depth, CTh cells with spontaneous firing were successfully found in L5 (Fig. $4 B, C$ ). Thalamic cells in VL, VA/VM, and Rt nuclei were identified by juxtacellular staining combined with calbindin and NeuN immunohistochemistry (Fig. $4 D-F$ ). Calbindin is more strongly expressed in VA/VM (Arai et al., 1994). In the following sections, we compared unit activities of four neuron subtypes during SWS and DS.

\section{Spike shape and burst firing patterns differ between CTh cells} and thalamic cell subtypes

Action potential kinetics (e.g., spike width) can differentiate neuron types (Tierney et al., 2004), with inhibitory neurons typically having narrower spikes than cortical pyramidal neurons. We compared the spike shape of CTh and thalamic neurons. The second phase of unit waves, which deflected in the negative direction, was narrower in the inhibitory Rt cells than in CTh or other thalamic neurons ( $p<0.05$; post hoc Tukey's test; Fig. $5 A, B)$, confirming that inhibitory thalamic cells have fast action potentials.

Thalamic cells can exhibit several fast spikes at short intervals (a burst) triggered by low threshold spikes (Deschênes et al., 1984). Therefore, we investigated the interspike intervals (ISIs) of thalamic cells in comparison with those of CTh cells. Some VL and VA/VM cells showed a bimodal distribution of ISIs with a first peak within $10 \mathrm{~ms}$ (Fig. $5 C$, top, burst spiking), whereas others showed a unimodal distribution (Fig. 5C, bottom, regular spiking) (Nowak et al., 2003). We calculated a "burst index" to quantify bursting behavior (see Materials and Methods) and found that bursting was significantly greater in thalamic cells than in CTh cells $(p<0.05$; Fig. 5D). Bursting in Rt cells, however, was lower than in VL and VA/VM relay cells $(p<$ 

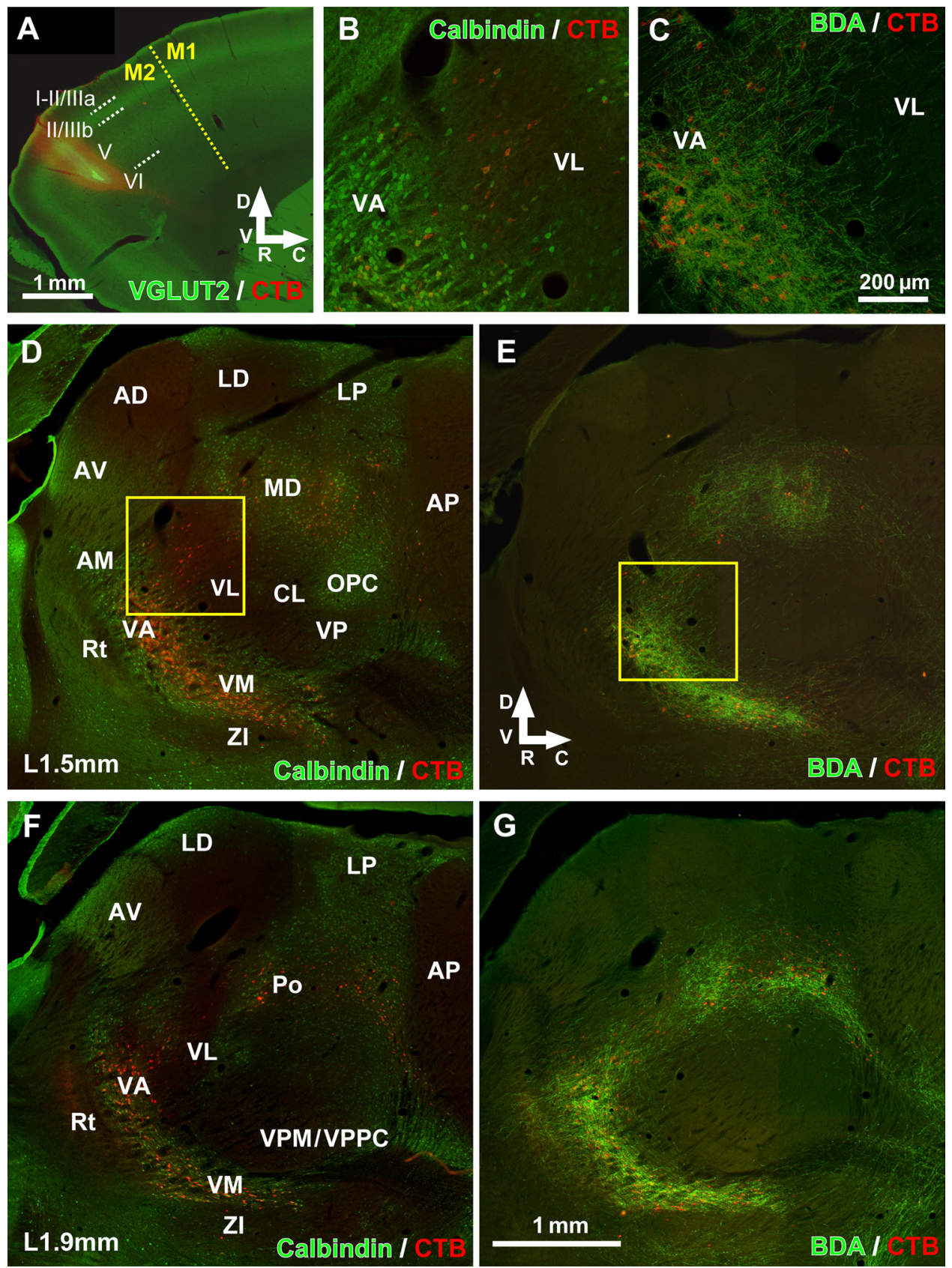

Figure 3. Reciprocal connections between the secondary motor area and thalamic VA/VM and VL nuclei. $A$, Deposit of a retrograde tracer (CTB; red) into secondary motor area (M2), combined with VGLUT2 immunofluorescence (green). An anterograde tracer (BDA) was simultaneously injected in the same location (data not shown). M1, Primary motor area. $\boldsymbol{B}$, Calbindin immunoreactivity much stronger in thalamic VA nucleus than in VL nucleus. Cells with red fluorescence, thalamic cells retrogradely labeled from M2. An enlargement of a rectangle in $\boldsymbol{D}$. C, Retrogradely labeled cells (red) and anterogradely labeled terminal fibers (green) in the thalamus. An enlargement of a rectangle in $\boldsymbol{E}$. $\boldsymbol{D}, \boldsymbol{E}$, Adjacent sagittal sections $1.5 \mathrm{~mm}$ lateral to midline. $\boldsymbol{F}, \boldsymbol{G}$, Adjacent sagittal sections $1.9 \mathrm{~mm}$ lateral to midline. Red fluorescent cells were labeled retrogradely from $\mathrm{M} 2$, with calbindin immunoreactivity (green) in $\boldsymbol{D}$ and $\boldsymbol{F}$, and with anterogradely labeled fibers (green) in $\boldsymbol{E}$ and $\boldsymbol{G}$. Note the dense distributions of retrogradely labeled cells in VA/VM and VL nuclei, and high spatial correlation between retrogradely labeled cells and anterogradely labeled terminal fibers. $A D$, Anterodorsal; $A M$, anteromedial; $A P$, anteroposterior; $A V$, anteroventral; $C L$, centrolateral; $L D$, laterodorsal; $L P$, lateroposterior; MD, mediodorsal; OPC, oval paracentral; Po, posterior; VP, ventroposterior; Zl, zona incerta.

0.01; Fig. 5D). Thus, burst patterns were different not only between pyramidal and thalamic cells but also between thalamic relay cells and Rt cells.

Firing frequency increase around Up-state onset differs between neuron subtypes

In vitro and in vivo data have suggested that the slow-wave oscillation results mainly from recurrent and balanced excitatory/inhibitory intracortical synaptic inputs, which partici- pate in Up states, and their absence, which may generate Down states (Steriade et al., 1993; Sanchez-Vives and McCormick, 2000). Yet the neuronal mechanisms that initiate Up states is not well understood. Recently, it has been suggested that thalamic neurons may participate in Up-state initiation (Crunelli and Hughes, 2010). If so, there should be increased activity in some thalamic neuron populations that precedes Up-state onset. To examine this, we compared the firing frequency change around Up-state start (US) in CTh and thalamic neurons. 

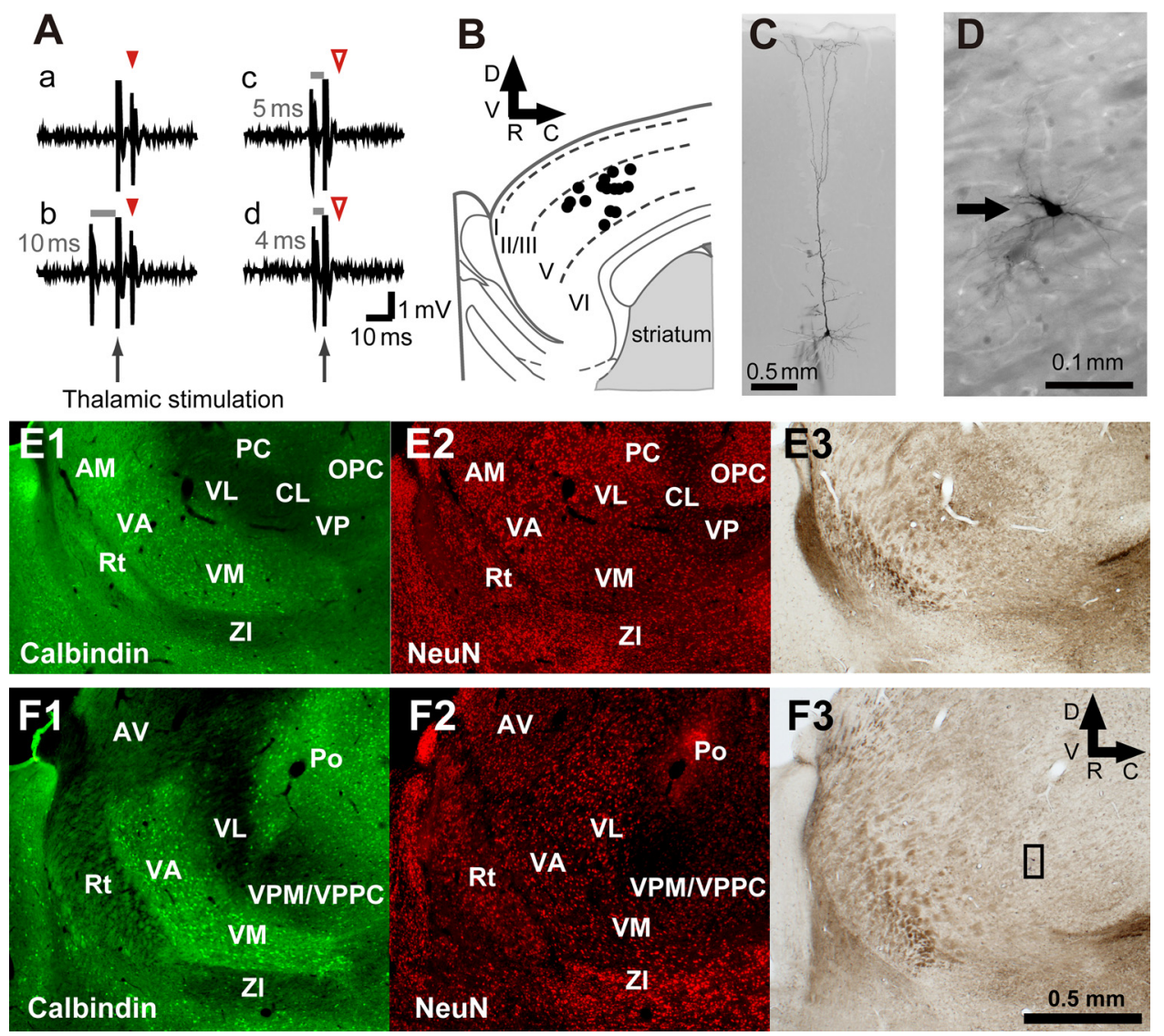

Figure 4. Cell subtype identification. $A$, Antidromic identification of CTh cell. This cell was antidromically activated by thalamic stimulation at 6 ms latency. Arrow, Thalamic electrical stimulation. $\boldsymbol{a}, \boldsymbol{b}$, Note stable latency of induced spikes (red arrowhead). $\boldsymbol{b}-\boldsymbol{d}$, Collision test. When spontaneous spikes occurred within $5 \mathrm{~ms}$ before the stimulation, antidromic spikes disappeared ( $\boldsymbol{c}, \boldsymbol{d}$, red open arrowhead). $\boldsymbol{B}$, Laminar location of (Th cells labeled with Neurobiotin. CTh cells distributed in layer 5 (L5) of the frontal cortex. D, Dorsal; V, ventral; $R$, rostral; $C$, caudal. C, CTh cell labeled with Neurobiotin. D, Thalamic cell labeled with Neurobiotin. This cell was situated in the VL nucleus. $\boldsymbol{E}$, Sagittal section (lateral $1.4 \mathrm{~mm}$ ) of thalamus stained for calbindin (E1, green) and NeuN (E2, red), and bright-field image (E3). Thalamic subregions were identified by these stainings. F, Another sagittal section but in lateral $1.9 \mathrm{~mm}$. F1, Calbindin (green). F2, NeuN (red). F3, Bright-field image. The area enclosed with rectangle (stained cell) was enlarged in $\boldsymbol{D}$.

After Up- and Down-state identification from the LFP (Fig. $6 A$ ), we made spike frequency histograms for individual units and determined the time from US at which spike generation occurred at the highest frequency (peak; Fig. 6C). Frequency peak points were different among the four neuronal subtypes $(p<0.01$; Fig. $6 B)$. Peaks were earlier in VA/VM and Rt cells than in CTh cells (Fig. $6 B$; CTh vs VA/VM, $p<0.01$; CTh vs Rt, $p<0.05)$.

To further quantify firing preference at the US, we obtained firing frequency at Up-state onset (from -50 to $+50 \mathrm{~ms}$ ) (Fig. $6 D)$ and US spike number ratio [(number of spikes between -100 and $+100 \mathrm{~ms}$ )/(total spike number)] (Fig. $6 E$ ). Firing frequency at Up-state onset was higher in VA/VM cells than in CTh cells $(p<0.05$; Fig. $6 D)$. Between thalamic subregions, there were cells firing at higher rates in VA/VM and Rt, but not in VL (Fig. $6 D$ ). The US spike number ratio was higher in Rt than in CTh cells $(p<0.01$; Fig. $6 E)$. These data suggest that more neurons in VA/VM and Rt selectively increase their firing at the transition from Down to Up state than do CTh neurons and neurons in VL.

\section{Temporal specificity of Up-state firing in CTh and thalamic cells}

If neuronal activity during Up states encodes specific information, there should be temporal firing preferences in individual neurons. To visualize temporal inhomogeneity of Up-state spikes, we made scatterplots of spikes with respect to start and end of Up states (Fig. $7 A$ ). Although Up-state durations were variable, we found temporal firing bias in some populations. Some neurons seemed to fire almost evenly within Up states but some others preferentially during the early or late half, as observed in both plots from all Up-state spikes (Fig. 7A, top) and from averaged times in individual Up states (Fig. $7 A$, bottom).

To compare firing patterns in Up states, we normalized each $\mathrm{Up}$-state duration to $1[-0.5$ (Up-state start) to 0.5 (Up-state end)] and obtained the normalized Up-state firing time for individual cells. The Up-state firing time was more variable in thalamic cells than in CTh cells (Fig. 7B). VA/VM cells tended to fire more spikes in the early, rather than late, half of Up states [Upstate firing time median: -0.13 for VA/VM $(p<0.01$; onesample $t$ test), -0.16 for Rt ( $p=0.05), 0.02$ for VL $(p=0.68)$, and 0.01 for CTh $(p=0.35)]$. VA/VM and Rt cells fired earlier in Up states than did CTh cells $(p<0.05)$. Thalamic cells firing earlier than -0.2 were mostly found in VA/VM ( 7 of 30 cells) and Rt (4 of 10 cells), but never in CTh (none of 24 cells) and only rarely VL neurons ( 1 of 12 cells) (Fig. $7 B, C$ ).

Thus, some neurons showed preferences for action potential generation in the early or late parts of Up states. If neurons fire during specific phases of Up states, the variance of firing times should increase considerably. To quantify temporal variability, we divided normalized Up states into five divisions (Fig. 7D). Some neurons fired uniformly across the five Up-state divisions, 
while others fired preferentially during specific divisions (Fig. 7D). To quantify temporal deviation in the five bin histograms of Up-state firing, we calculated $\chi^{2}$ values and tested their significance using the $\chi^{2}$ test for goodness of fit (Fig. 7E) (see Materials and Methods). Larger $\chi^{2}$ values indicate higher variances of spike numbers among five bins, suggesting activity concentration in specific Up-state subdivisions (Fig. $7 D ; \chi^{2}$ value 9.49 corresponds to $p=0.05)$. The $\chi^{2}$ values of CTh (median, 16.9) and VL cells (median, 23.3) were relatively small, but those of VA/VM (median, 36.7) and Rt cells (median, 72.5) were larger (Fig. 7E; $p<0.05$, ANOVA; $p<0.05$ in CTh vs VA/VM). There were more cells with larger $\chi^{2}$ values in VA/VM and Rt than in CTh and VL $(p<0.05$; Kolmogorov-Smirnov test; Fig. $7 F)$.

The median firing times of CTh cells were distributed mostly around the center of the Up state, but those of thalamic cells varied widely within Up states (Fig. $7 B$ ). We investigated the relationship of firing times with $\chi^{2}$ values. $\chi^{2}$ values of thalamic cells were larger in cells with early Upstate firing time than those with late firing $(p<0.05$; Fig. $7 G$, right), but this difference was not found in CTh cells (Fig. 7G, left). These observations indicate that CTh cells fire more uniformly during Up states, while thalamic cells, especially $\mathrm{VA} / \mathrm{VM}$ and Rt neurons fire preferentially at a given Up-state phase, particularly in early to middle phases.

\section{Temporal specificity of Down-state firing in thalamic cells}

In contrast to CTh cells, some thalamic neurons fired not only in Up but also in Down state [ratio of spike number in Down state to that in whole SWS: $0.19 \pm 0.11 \mathrm{in} \mathrm{VL}(n=12), 0.18 \pm 0.15$ in $\mathrm{VA} / \mathrm{VM}(n=30), 0.12 \pm 0.09$ in Rt $(n=10)$, and $0.07 \pm 0.07$ in CTh $(n=24)]$. This suggests that thalamic cells may exhibit functional activity during Down states in addition to Up states, and be differentiated according to the Down-state phase. To specify Down-state firing times, each Down-state duration was normalized to 1 [ -0.5 (Down-state start) to 0.5 (Down-state end)]. The median of normalized firing times was obtained for each neuron in VL, VA/VM, and Rt (Fig. 8A,B). VA/VM and Rt cells fired later in Down state, but VL cells did not [Down-state firing time median: 0.22 for VA/VM $(p<0.01$; one-sample $t$ test), 0.35 for Rt $(p<0.05)$, and 0.14 for VL $(p=0.34)$ ]. To further quantify firing time specificity during Down states, we divided the normalized Down states into five divisions and obtained the $\chi^{2}$ values. Thalamic cells showing preferred Downstate firing times across the five Down-state divisions [values of $\chi^{2}>9.49(p=0.05)$ ] were relatively abundant in VA/VM (19 of 27 cells) and Rt ( 8 of 9 cells), but were less frequent in VL ( 5 of 12 cells) (Rt vs VL, $p<0.05, \chi^{2}$ test; Fig. $8 C$, bottom).

To confirm temporal firing preferences during late-Down states in VA/VM and Rt cells, we investigated the relationship of

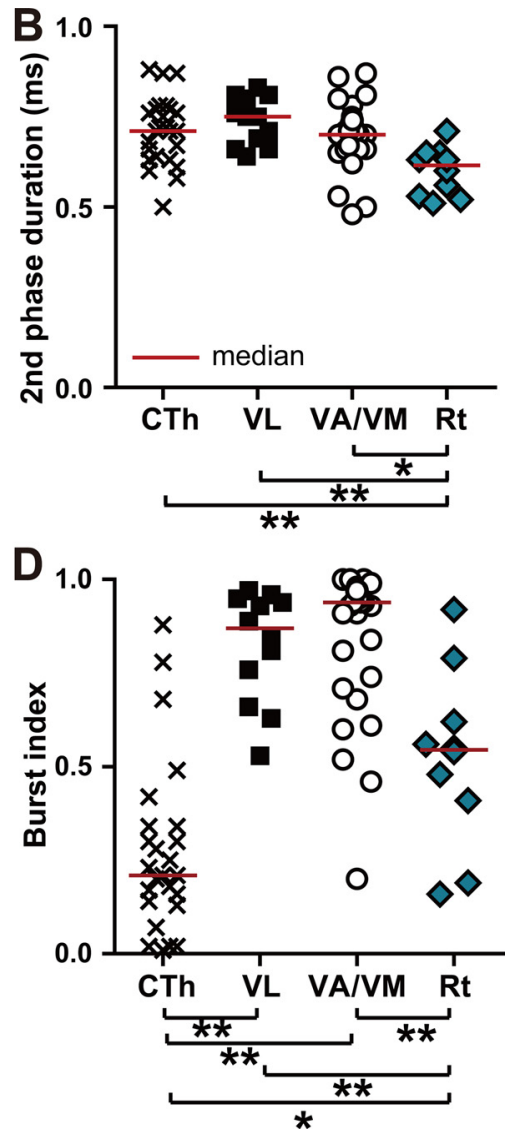

Figure 5. Spike shape and burst firing pattern were different between CTh cells and thalamic cell subtypes. $\boldsymbol{A}$, Spike waveforms terval $<200 \mathrm{~ms}$ ). Those of (Th cells were smaller than those of thalamic subtypes. Between thalamic subregions, Rt cells had smaller burst indices. ${ }^{* *} p<0.01 ;{ }^{*} p<0.05$; post hoc Tukey's test.

$\chi^{2}$ values with Down-state firing time (Fig. $8 D$ ). $\chi^{2}$ values were larger in VA/VM and Rt cells at later Down-state times [correlation coefficient (c.c.) $=0.37$ in VA/VM; c.c. $=0.33$ in Rt] (Fig. $8 D$ ). The regression slope was significantly different between VL and Rt cells ( $p<0.01$, comparison of two regression parameters). This demonstrates that VA/VM and Rt neurons preferentially fire near the end of Down states.

\section{Firing specificity during Up/Down cycles}

Some VA/VM and Rt cells preferentially fired early in Up states (Fig. 7) or late in Down states (Fig. 8). We therefore investigated the firing specificity during combined cycles of normalized Up and Down states. Some neurons showed firing peaks during combined Up/ Down cycles (Fig. 9A). We investigated whether spiking behavior was significantly modulated during combined Up/Down cycles, using circular analysis. Individual Down- and Up-state spike histograms were transformed into circular representations $\left[0^{\circ}\right.$, Downstate start (Up-state end); $90^{\circ}$, Down-state center; $180^{\circ}$, Down-state end (Up-state start); $270^{\circ}, \mathrm{Up}$-state center]. To estimate the modulation phase and intensity, the mean vector (mean angle and angular concentration) was calculated for individual neurons (Fig. 9B, modulation phase of $260^{\circ}$ for $\mathrm{CTh}, 319^{\circ}$ for VL, $165^{\circ}$ for VA/VM, $216^{\circ}$ for $\mathrm{Rt}$ in these cases). Angular concentrations varied inversely with the amount of dispersion and ranged from 0 (uniform distribution) to 1 

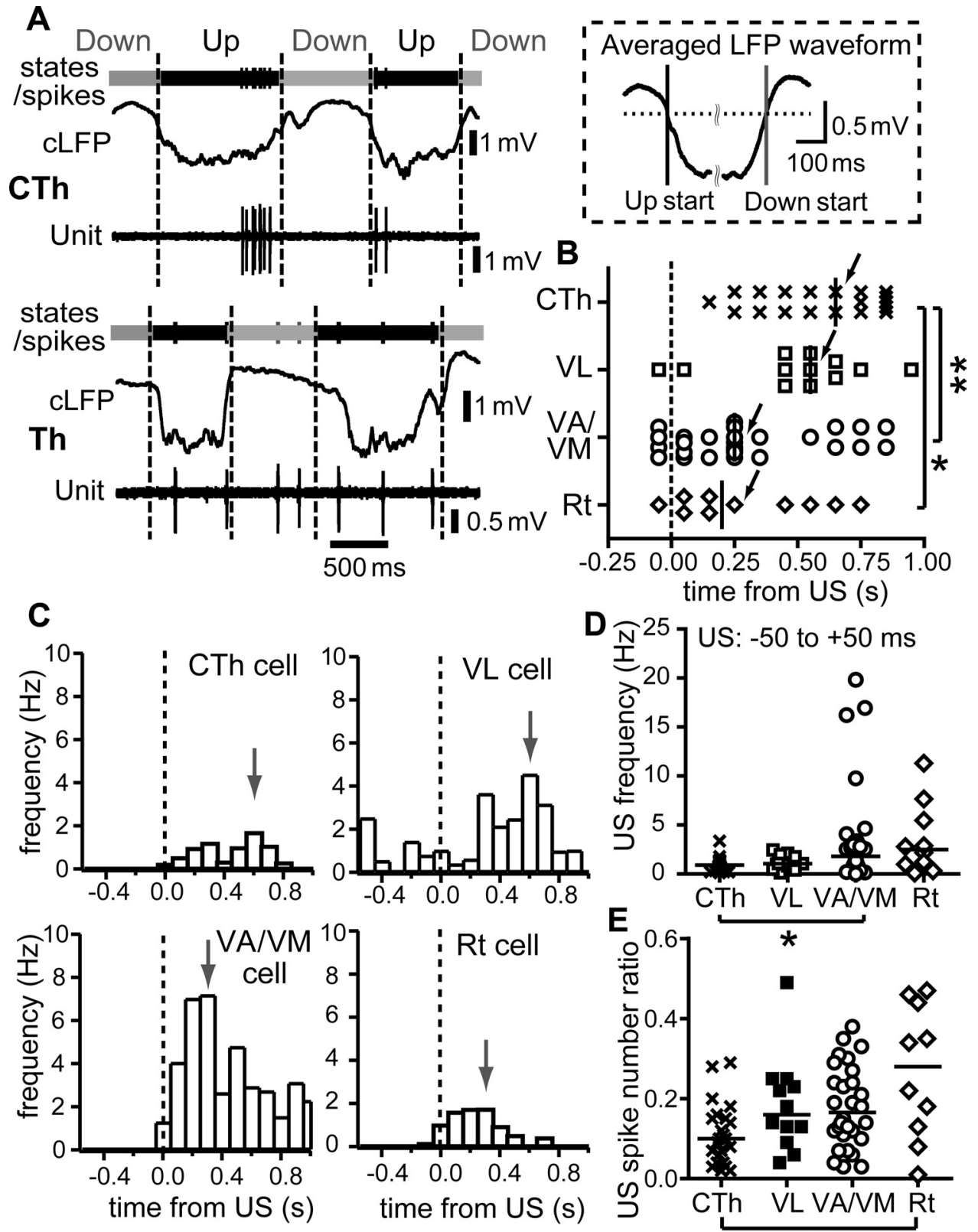

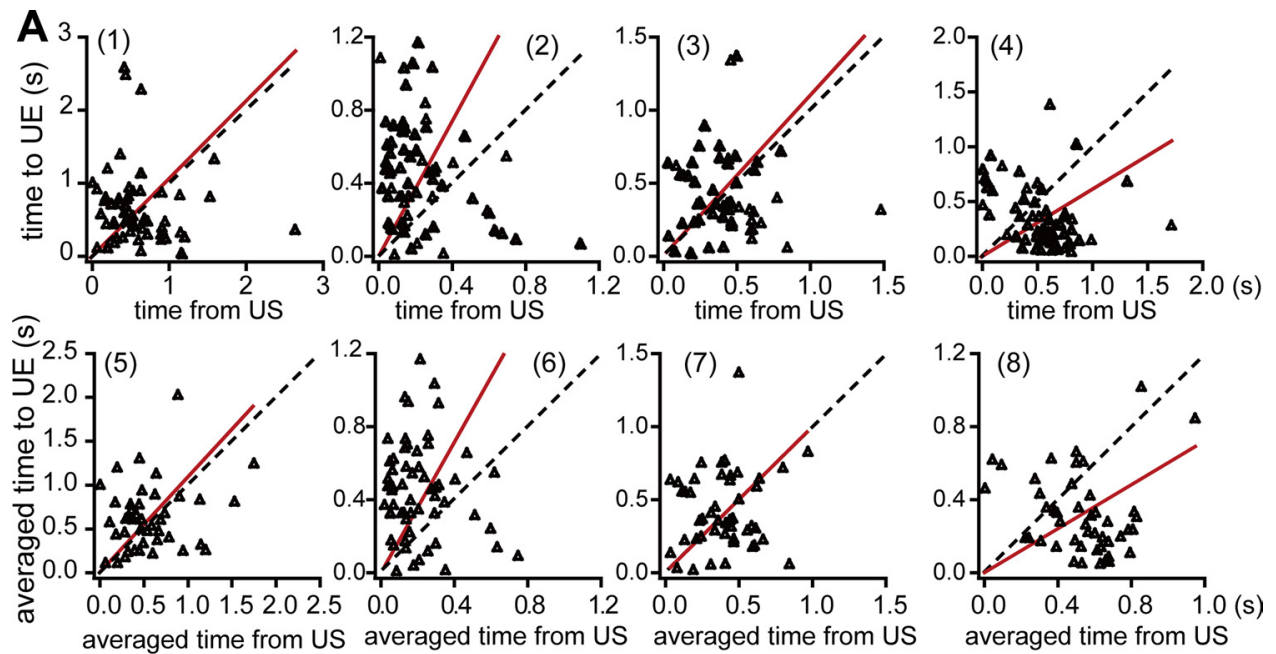

B
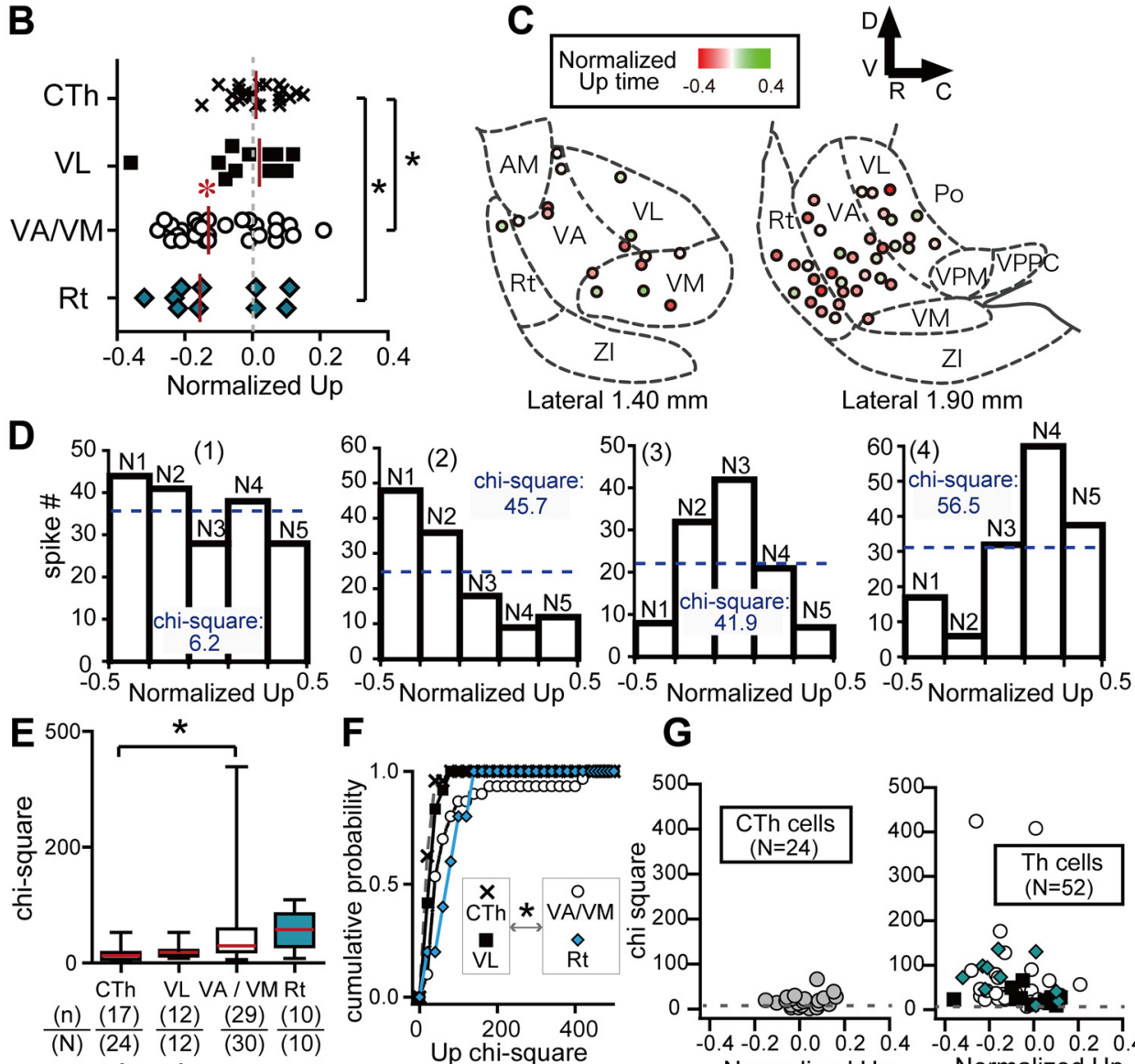

G

$$
\text { (N) } \underbrace{\underbrace{\dagger}}_{\dagger \dagger} \text { n: no. of significantly deviated cells }
$$

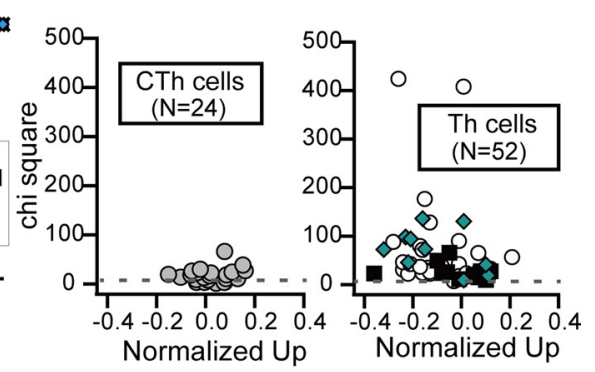

Figure 7. Firing time specificity during Up state. A1-A4, Scatterplots of the spikes discharged within Up states with respect to temporal differences from start and to end of Up states (US and UE, respectively; obtained from four VA/VM cells). Red line, Averaged slope of each spike point; dashed line, center of Up state. A5-A8, Same configuration as in above but spikes within each Up state were averaged. Note that some neurons preferentially fired during early $(\boldsymbol{A} \mathbf{2}, \boldsymbol{A} \mathbf{6})$ or late half $(\boldsymbol{A 4}, \boldsymbol{A} \boldsymbol{8})$ of Up states. Up-state duration was $>40 \mathrm{~ms}$. $\boldsymbol{B}$, Firing times in normalized Up states different among the four subtypes $(p<0.01)$. VA/VM cells fired in the early half of Up state $\left(p<0.01\right.$; one-sample $t$ test). VA/VM and Rt cells fired earlier in Up states than CTh cells $\left({ }^{*} p<0.05\right)$. Vertical red line, Median; dashed line, Up-state center. C, Normalized Up-state firing times in thalamic subregions, shown using a color code scale from red $(-0.4)$ to green $(0.4)$. D, Dorsal; V, ventral; R, rostral; C, caudal. Note cells with firing in early Up-state half are more found in VA/VM and Rt. AM, Anteromedial nucleus; Po, posterior thalamic nuclear group; VPM, ventral posteromedial thalamic nucleus; VPPC, ventral posterior nucleus-parvicellular part; Zl, zona incerta. D, Spike timing variability during normalized Up states. $\chi^{2}$ test for goodness of fit was applied to the firing time distribution (5 bins). D1, Uniform distribution. D2-D4, Significantly deviated from the uniform distribution; early-, center-, and late-peaked cells, respectively. D1-D4 correspond to neuron types shown in A1-A4. Dotted line, Mean spike number. $\boldsymbol{E}, \chi^{2}$ values in Up state different among four groups (boxplots; $\left.p<0.05\right)$. Those of VA/VM cells were larger than those of (Th cells $\left({ }^{*} p<0.05\right.$ ). Horizontal red line, Median; box, 25th to 75th percentile; whiskers, data range. Bottom, [(Number of significantly deviated cells)/(total cell number)] in individual subtypes. Modulated proportion in CTh cells was smaller than that in VA/VM $\left({ }^{\dagger \dagger} p<0.01 ; \chi^{2}\right.$ test) or VL cells $\left({ }^{\dagger} p<0.05\right)$. $\boldsymbol{F}$, Cumulative plot for $\chi^{2}$ values. VA/VM and Rt cells were significantly different from CTh and VL cells $(p<$ 0.05 ; Kolmogorov-Smirnov test). G, Relationship of $\chi^{2}$ values (temporal specificity) with normalized Up-state firing times. Thalamic cells showed more temporally specific firings in Up states than cortical pyramidal cells. Dotted line, $\chi^{2}$ value of $9.49\left(p=0.05\right.$ in $\chi^{2}$ test for goodness of fit). 
A

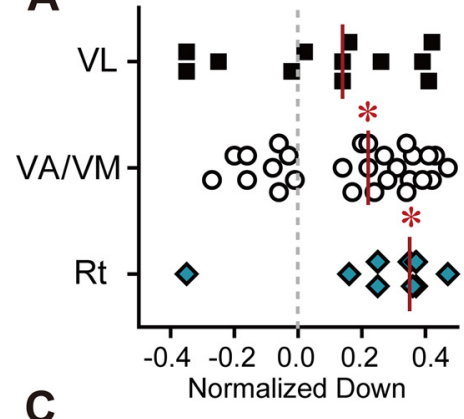

C

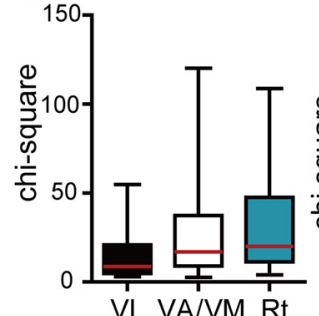

$\frac{(\mathrm{n})}{(\mathrm{N})} \frac{(5)}{(12)} \frac{(19)}{(27)} \frac{(8)}{(9)}$

$\dagger \mathrm{n}$ : no. of significantly deviated cells

D

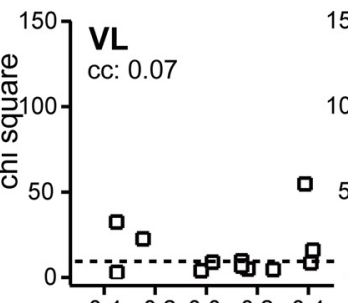

Normalized Down

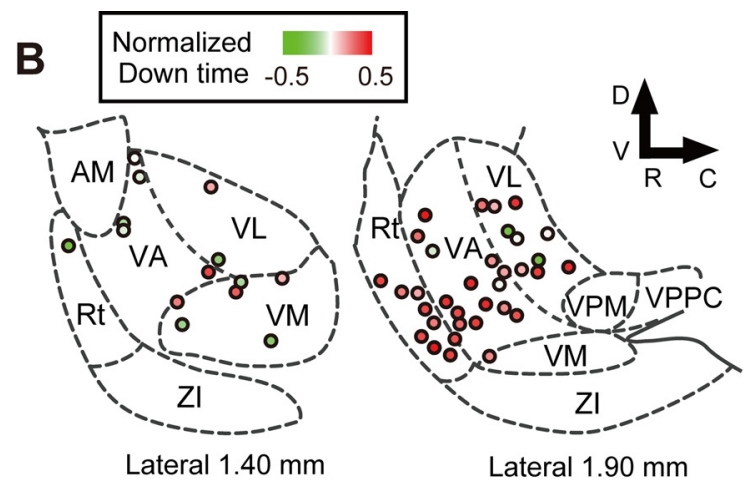

$\begin{array}{llllllll}-0.4 & -0.2 & 0.0 & 0.2 & 0.4\end{array}$

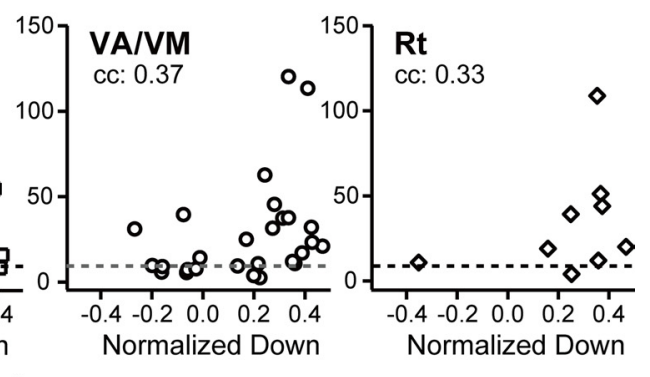

Figure 8. Firing time specificity of thalamic cells during Down state. $A$, Firing times in normalized Down states. Those of VA/VM and Rt cells were biased to the late half $(p<0.01$ in VA/VM; $p<$ 0.05 in Rt cells), while VL cells did not show significant bias in firing timing. Vertical red line, Median; dashed line, Down-state center. $\boldsymbol{B}$, Normalized Down-state firing times in thalamic subregions, shown using a color code scale from green $(-0.5)$ to red (0.5). D, Dorsal; V, ventral; R, rostral; $C$, caudal. Note cells with firing in late half of Down state distributed densely in VA/VM and Rt. $C$, Down-state $\chi^{2}$ values, shown in the same configuration as in Figure 7E. Larger values were found in VA/VM and Rt, but the values not different among the three thalamic subregions $(p=0.24$; ANOVA). Bottom, [(Number of significantly deviated cells)/(total cell number)] in individual subtypes. Modulated proportion of Rt cells was larger than that of $\mathrm{VL}$ cells $\left({ }^{\dagger} p<0.05 ; \chi^{2}\right.$ test). $\boldsymbol{D}$, Relationship of $\chi^{2}$ values with normalized Down-state firing times of thalamic cells. Some VA/VM and Rt cells fired selectively around the end of Down states, but VL cells did more uniformly. Dotted line, $\chi^{2}$ value of 9.49 ( $p=0.05$ in $\chi^{2}$ test for goodness of fit).

$10 A b)$. The first peak was $0.12 \pm 0.03(n=7)$, compatible with the spindle rhythm.

Then, we asked whether CTh and thalamic cell firings were modulated by spindles, using the spindle waves recorded in deep cortical layers (mean cycle interval, $0.10 \pm 0.002 \mathrm{~s} ; n=4$ ). Negative troughs of spindles were defined as $0^{\circ}$. Some CTh and thalamic cell firings were modulated by spindle cycles (Fig. $10 \mathrm{~B}$ ). To examine preferred firing phase and modulation strength, we quantified the mean angle and normalized length (angular concentration) of vectors, using circular analysis. The angular concentration was different among the four neuronal subtypes $(p<0.01$; ANOVA; Fig. 10C). Rt cells had larger angular concentrations than the other neuron subtypes $(p<0.01$ in CTh vs Rt and VL vs Rt; $p<0.05$ in VA/VM vs Rt; Fig. 10C). Spindlemodulated cells were identified by Rayleigh test $(p<0.05)$ [ (modulated cell number)/(total cell number), shown in the bottom of Figure 10C]. The modulated cell proportion was lower in VL than in Rt cells $\left(p<0.05 ; \chi^{2}\right.$ test $)$.

Next, the mean angle and angular concentration were compared among spindle-modulated cells of the four subtypes (Fig. $10 \mathrm{D})$. The mean angle of modulated cells was $14^{\circ}$ for CTh, $229^{\circ}$ for $\mathrm{VL}, 211^{\circ}$ for $\mathrm{VA} / \mathrm{VM}$, and $188^{\circ}$ for Rt cells (negative trough, $0^{\circ}$ ) (Fig. $10 \mathrm{E}$ ). The mean angular concentration of modulated cells (vectorial sum) was 0.05 for CTh, 0.09 for VL, 0.13 for VA/ VM, and 0.24 for Rt cells (Fig. 10D). These suggest that thalamic cells are more strongly modulated during a spindle than are CTh cells. VA/VM and Rt cells had preferred firing angles (Fig. 10E; $p<0.05$, Hodges-Ajne test for circular uniformity). The modulated phase was different among the four subtypes $(p<0.05$; four-sample Watson-Williams test). CTh cells were different from VA/VM, Rt cells ( $p<0.01)$, and VL cells ( $p<0.05$; twosample Watson-Williams test). These observations suggest differences among neuron subtypes in spindle modulation strength and firing timing during spindle cycles.

Since VA/VM thalamic cells mainly innervate L1a and VL cells do L2/3b, spindle-modulated excitations would generate current sinks within superficial layers, with current sources in deeper layers. Therefore, spindle phase would be expected to reverse between superficial and deep layers. We examined this point by comparing spindle LFPs at various cortical depths (Fig. 11A,B). Up states reversed phase in the middle layer, with negativities with higher frequency components found in L5 (Fig. 11A,C). Spindle waves also reversed in middle layers (Fig. $11 A, C, D)$. The reversal of LFPs was confirmed in three rats.

\section{Neuron subtype-dependent firing preference between SWS and DS}

Slow waves are observed in sleeping and anesthetized states, but desynchronizes upon waking. It is conceivable that the firing patterns of cortical and thalamic neurons are dependent on these brain states. To clarify state-dependent firing changes, we continuously recorded unit activity in both SWS and DS for individual cells (Fig. 12A, top and bottom, respectively) and compared their firing frequency in these two states. Some neurons showed higher firing frequency in SWS than in DS (SWS-active; Fig. 12A, left), but others had higher frequencies in DS (DS-active; Fig. 12A, right). Overall firing frequencies in SWS were not different among cell types $(p>0.05)$, but firing frequencies in DS were neuron subtype specific (Table $1 ; p<0.05$; ANOVA). Firing frequencies decreased during the transition from SWS to DS in 

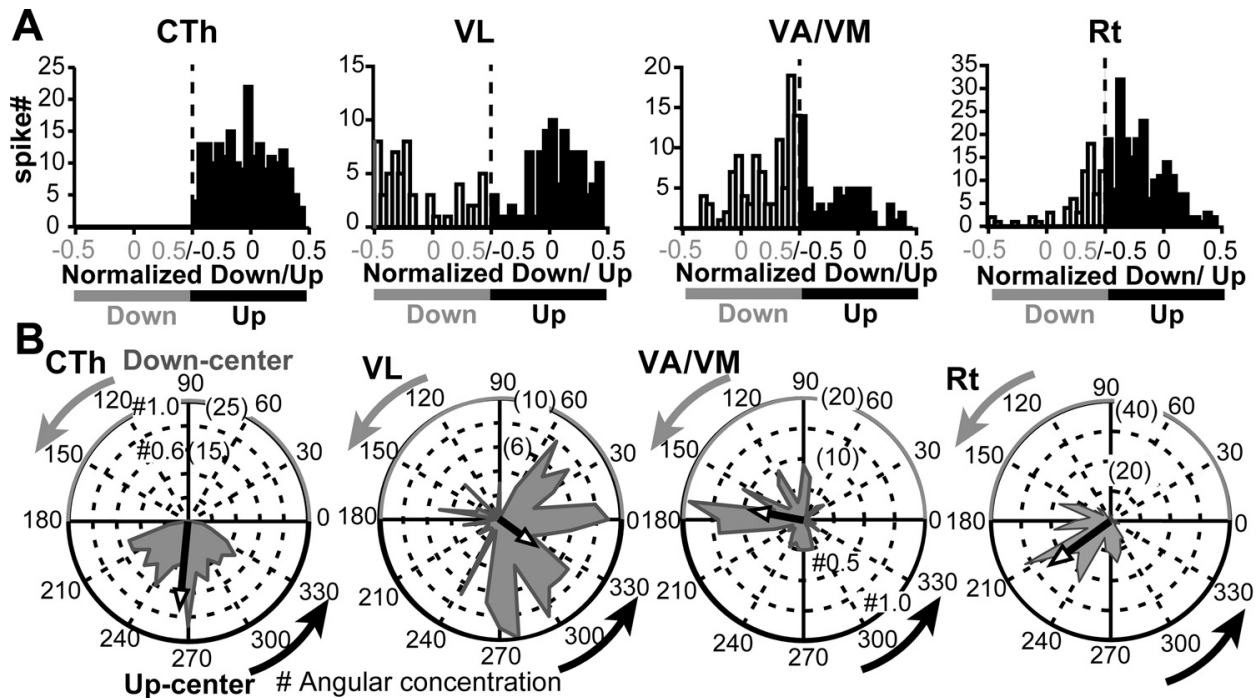

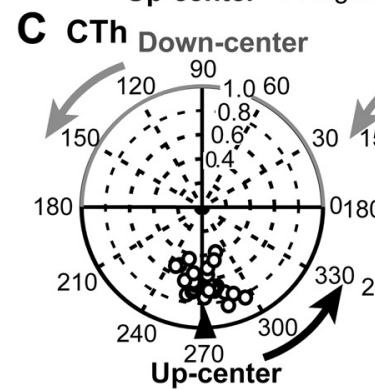

D

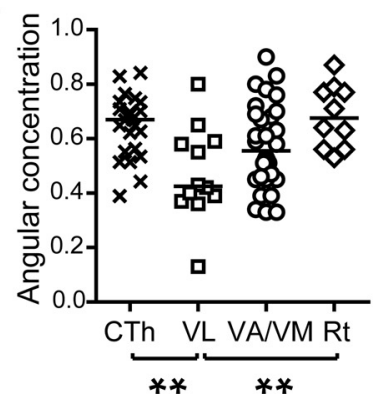

VL
VA/VM

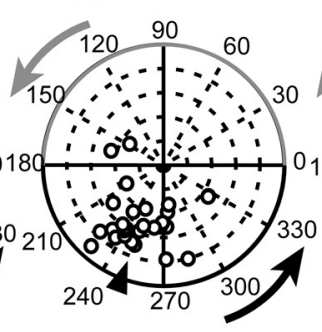

Rt

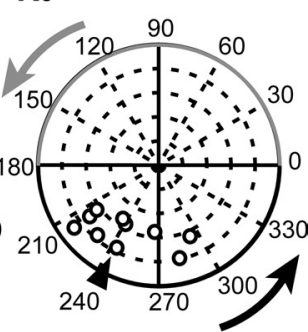

E

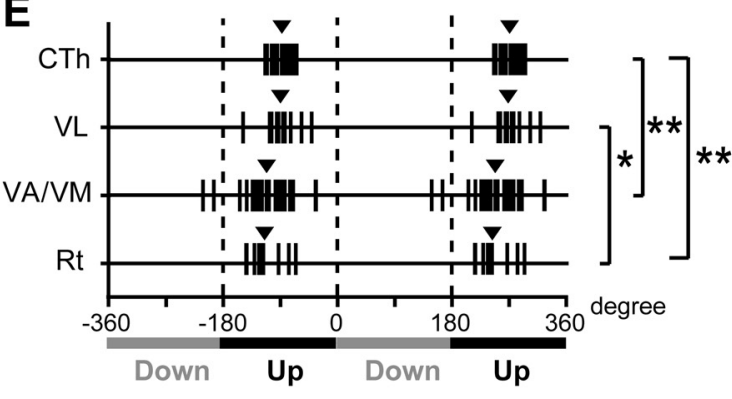

Figure 9. Heterogeneous firing phases in Up/Down cycles. $A$, Firing time distribution of four cells of different groups during normalized Down and Up state. Individual state durations were normalized to 1 [ -0.5 , state start; 0.5 , state end, respectively]. Note diversity in peak and dispersion of firing times among neurons. $\boldsymbol{B}$, Circular representation of firing times during Up/Down cycles transformed from the spike histograms shown in $\boldsymbol{A}\left[0^{\circ}\right.$, Down-state start (Up-state end); $90^{\circ}$, Down-state center; $180^{\circ}$, Down-state end (Up-state start); $270^{\circ}$, Up-state center]. Radius, Spike number in each bin. (n), Spike number. Arrow, Mean vector. \#, Angular concentration. C, Firing phase preference in CTh and thalamic cells. Radius, Angular concentration. Open circle, Preferred angle and angular concentration in each cell; arrowhead, angle of mean vector in each subtype. $\boldsymbol{D}$, Angular concentrations of $(T$ h and thalamic cells. Preference intensity was different among four subtypes $\left(p<0.01 ;\right.$ ANOVA). CTh and Rt cells had larger angular concentration than VL cells $\left({ }^{* *} p<0.01\right)$. Horizontal lines, Median values. $E$, Preferred firing angles in circular analysis, different among four subtypes ( $p<0.01$; four-sample Watson-Williams test). Preferred phases in (Th cells were different from those in VA/VM and Rt cells $(p<0.01)$, and those were also different between VL and Rt cells ( $p<0.05$; two-sample Watson-Williams test). Arrowhead, Mean angle of individual subtypes. Cycles are shown twice for clarity.

VA/VM cells, but increased in VL cells (Table $1 ; p<0.05$ and $p<$ 0.01 , respectively). To quantify firing frequency changes between SWS and DS, we calculated the DS preference of firing (Fig. $12 B, D)$ (see Materials and Methods) (values from -1 to $1: 1$, DS-only activity; -1 , SWS-only activity). VA/VM cells fired preferentially in SWS ( $p<0.05$; one-sample $t$ test), while VL cells preferred to fire in DS $(p<0.01)$. In contrast, CTh and Rt cells were diverse in DS preference and did not show a clear firing preference for either state ( $p=0.09$ and $p=0.80$, respectively). DS preference was higher in VL cells than in CTh or VA/VM cells $\left({ }^{* *} p<0.01\right)$.

The slow wave is composed of Up-state depolarizations and Down-state hyperpolarizations, whereas DS is characterized by continuous depolarization. Up depolarizations may be a tempo- rally restricted fragment of DS (Destexhe et al., 2007). If so, firing pattern differences between neuron subtypes should be preserved in both the Up states and DSs. To shed light on this question, we compared the firing frequency between the Up states and DSs in individual subtypes, using DS preference compared with Up state (1, DS-only activity; -1 , Up-only activity). Up-state firing frequency was not different among subtypes (Table $1 ; p>0.05$; ANOVA). CTh and VA/VM cells fired preferentially in the Up states $(p<0.01$; one-sample $t$ test), while VL cells preferred to fire in DS $(p<0.01)$. Rt cells did not show clear significant preference in relative Upstate/DS firing $(p=0.46)$. DS preference compared with Up states was higher in VL cells than the other subtypes (Fig. $12 C$; ${ }^{* *} p<0.01$ in CTh vs VL and VA/VM vs VL; ${ }^{\star} p<0.05$ in Rt vs VL). These suggest that state dependency of spike activity differs between CTh 
A

(a)<smiles>[134I]</smiles>

\section{LFP}

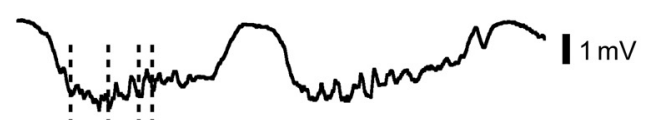

Spindle

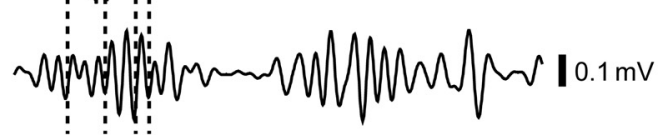

Unit

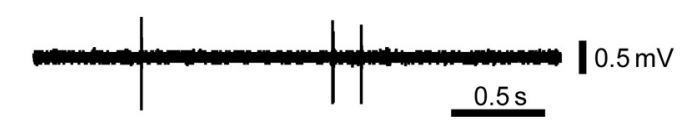

B

B CTh cell
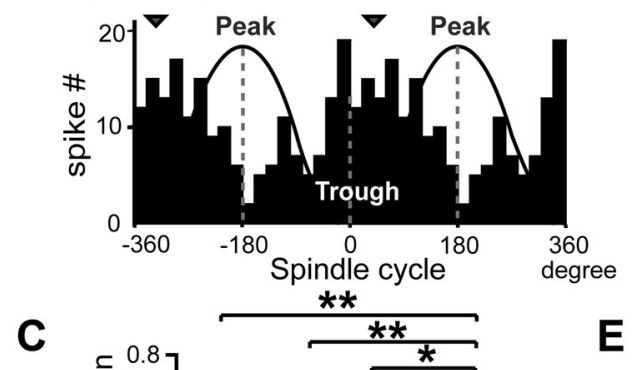

C
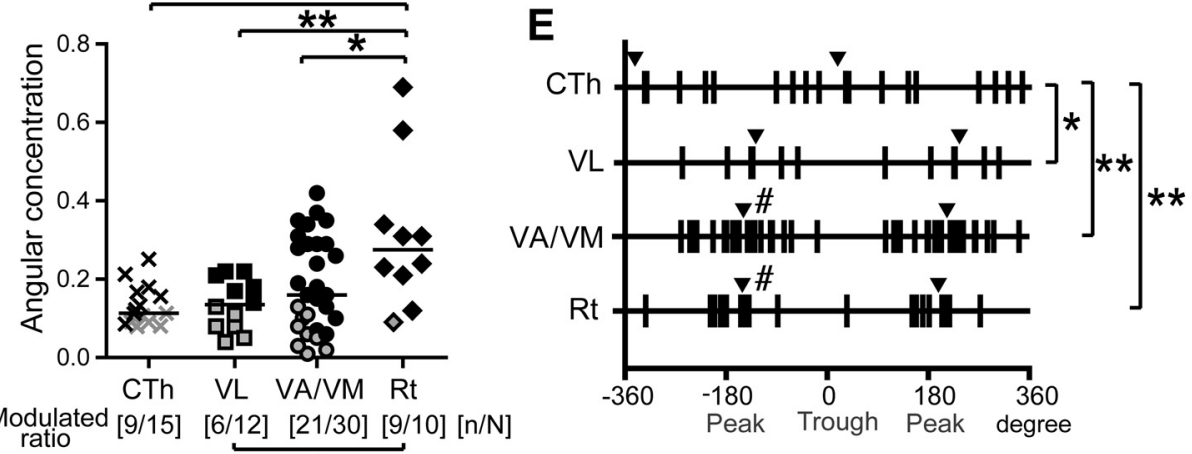

D CTh

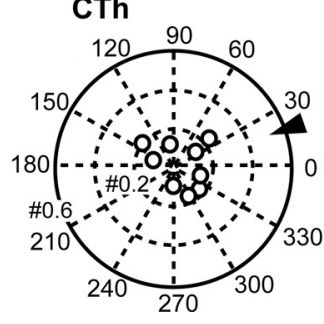

VL

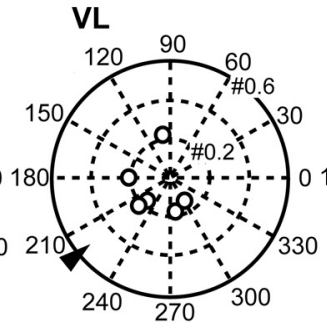

(b)

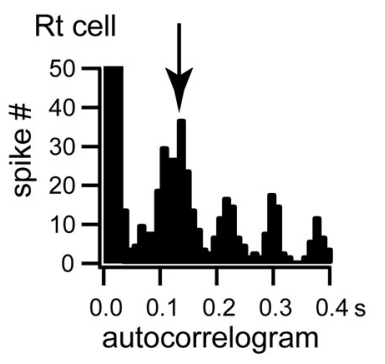

VA/VM cell

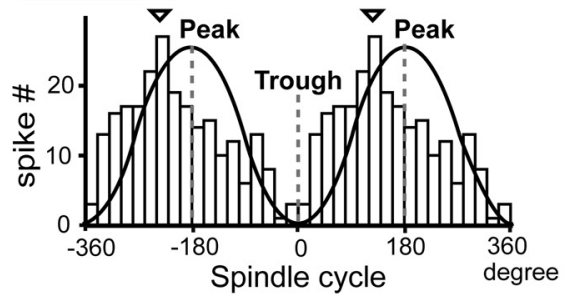

E

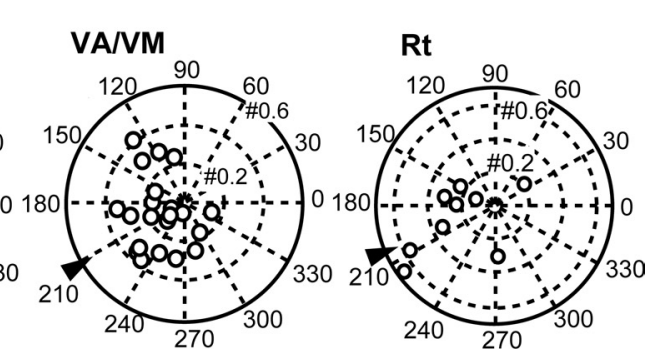

Figure 10. Spindle modulation of CTh and thalamic cell firing. Aa, Spindle waves during slow wave Up states. Spindle waves were prominent in Up states and disappeared in Down states. The troughs of spindles obtained by filtering corresponded to those on the Up state of the raw LFP. $\boldsymbol{A} \boldsymbol{b}$, Autocorrelogram of a Rt cell firing. Note prominent peak around $0.1 \mathrm{~s}$, which is in spindle band range. $\boldsymbol{B}$, Spike distribution of CTh and VA/VM cells during spindle cycles. Arrowheads point to the mean firing phase. Some (Th cells and thalamic cells discharged spikes in rhythm to the spindle oscillation during Up states. $C$, Angular concentrations of (Th and thalamic cells, different among four subtypes $\left(p<0.01\right.$; ANOVA). Those in Rt cells were larger than those in other subtypes $\left(* * p<0.01\right.$; ${ }^{*} p<0.05$; posthoc Tukey's test). Horizontal lines, Median values. Thicker marks, Cells modulated significantly ( $p<0.05$; Rayleigh's test). Bottom, [(Number of significantly spindle-modulated cells)/(total cell number)] in individual subtypes. Modulated proportion was different between VLand Rt cells $\left({ }^{\dagger} p<0.05 ; \chi^{2}\right.$ test). $\boldsymbol{D}$, Modulation intensity and firing phase of modulated cells in the spindle. Arrowhead, Angle of mean vector. $\boldsymbol{E}$, Firing angles of modulated cells in circular analysis. The preferred angles were different between (Th cells and VL or VA/VM or Rt cells $\left(* * p<0.01\right.$; ${ }^{*} p<0.05$; two-sample Watson-Williams test). Arrowhead, Mean angle of individual subtypes. Cycles are shown twice for clarity. "Significantly preferred firing angles ( $p<0.05$; Hodges-Ajne test).

cells and thalamic subtypes, and that Up states and DS are not simply equivalent depolarized states.

\section{Discussion}

To understand (1) the neural activity around the Up-state onset, (2) temporal specificity of firing during Up states or spindle waves, and (3) differences between Up state and DS, we analyzed neural activity in vivo during SWS and DS in L5 CTh cells and thalamic neurons from three regions: VL, receiving input from cerebellum; VA/VM, receiving input from the basal ganglia; and Rt, which inhibits the dorsal thalamus (Table 2). Preferential firing around Up-state initiation was found more frequently in VA/VM and Rt cells than in CTh and VL cells. Greater temporal specificity of Up-state firing was found in thalamic cells than in CTh cells. Firing coupling with the spindle was stronger in Rt cells, and the coupling phase was different between thalamic and $\mathrm{CTh}$ cells. Firing frequency during Up states was higher in VA/VM cells and lower in VL cells than that in DS. These findings revealed that cortico-thalamo-cortical subnetworks are temporally differentiated during slow and spindle oscillations, and that Up states and DS are different types of depolarized states. 
A

LFP

Spindle

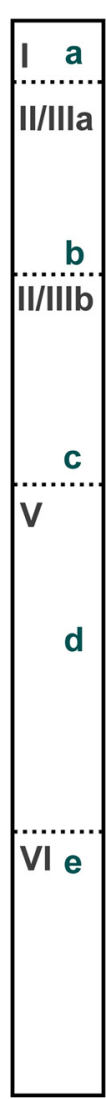

a ref.

Yalor Dumar

inu $(100 \mu \mathrm{m})$

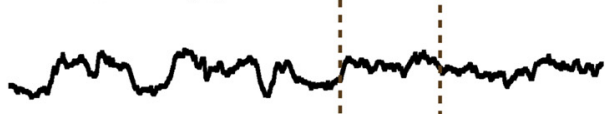

- .

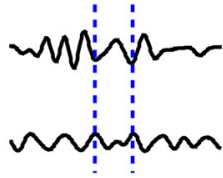

b ref.

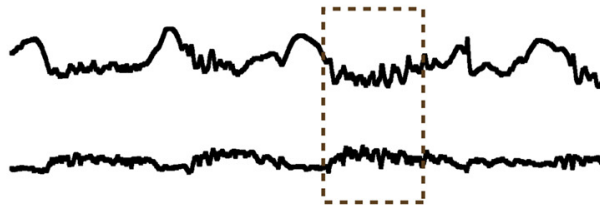

miniving $(400 \mu \mathrm{m})$

(1)

C ref

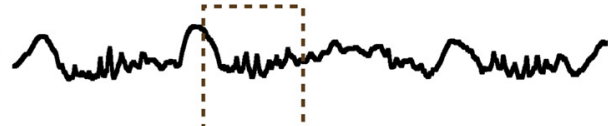

$(600 \mu \mathrm{m})$

minin

molnm

MWMM

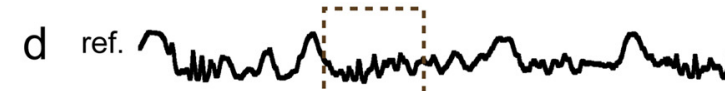

$(1000 \mu \mathrm{m})$

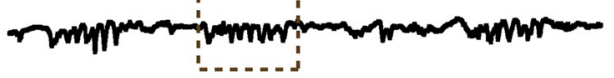

ninting

niniman

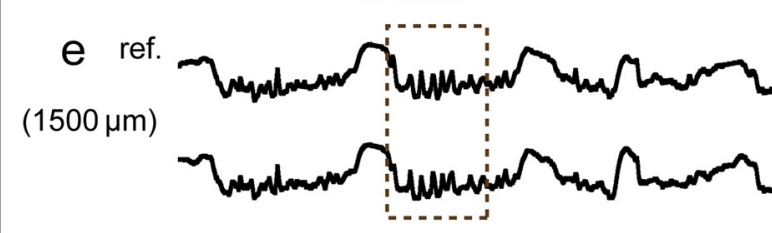

winimer :

wionsh

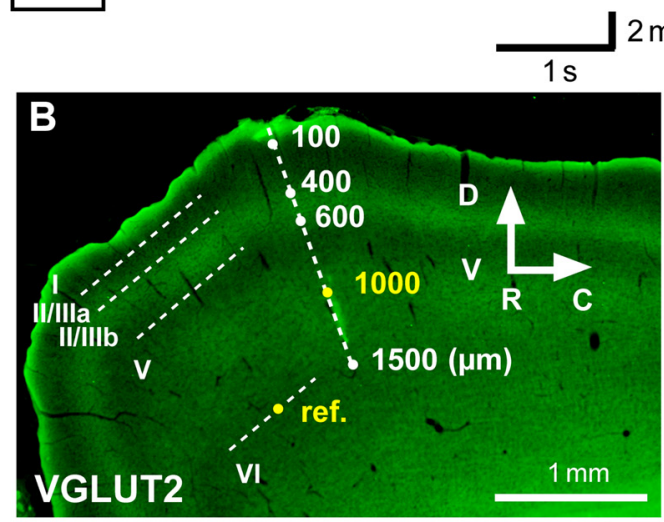

$0.5 \mathrm{~s} 0.4 \mathrm{mV}$

D
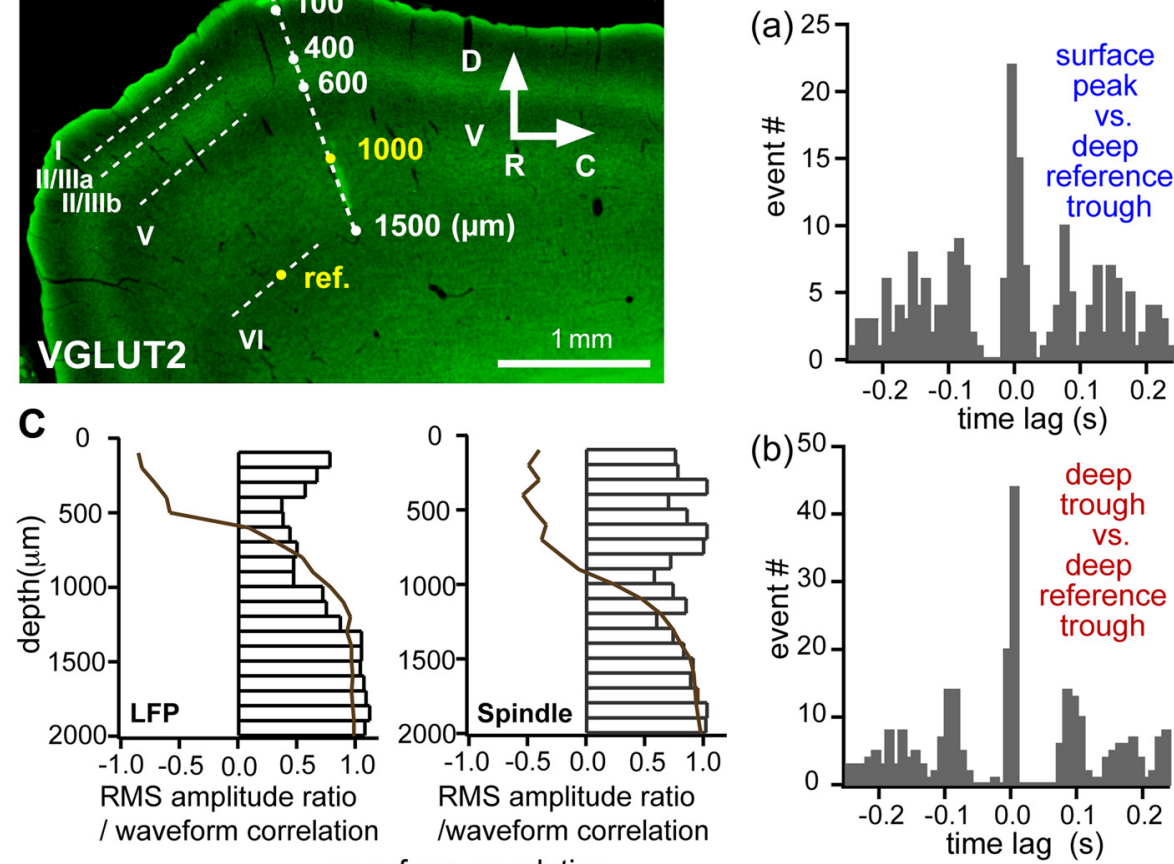

$\square$
$\square$ RMS amplitude ratio

Figure 11. Cortical depth dependency of slow-wave and spindle oscillations. A, LFPs recorded simultaneously at two different depths. One electrode was placed at the deepest as a reference (ref.), but the other was moved from superficial to deeper layers $(\boldsymbol{a}, 100 \mu \mathrm{m}$ deep; $\boldsymbol{b}, 400 \mu \mathrm{m} ; \boldsymbol{c}, 600 \mu \mathrm{m} ; \boldsymbol{d}, 1000 \mu \mathrm{m} ; \boldsymbol{e}, 1500 \mu \mathrm{m})$. Slow waves and spindles recorded in the superficial layers (L1 and L2/3) negatively correlated with those in the deepest reference [raw LFP (slow wave) correlation: -0.85 in $\boldsymbol{a}$ and -0.61 in $\boldsymbol{b}$; spindle correlation: -0.40 in $\boldsymbol{a}$ and -0.54 in $\boldsymbol{b}$; see blue dashed lines]. In middle layers, correlations of both waves became weaker (slow wave and spindle correlation in $c: 0.09$ and -0.34 , respectively). In L5, positive correlations were found (slow wave correlation: 0.79 in $\boldsymbol{d}$ and 0.97 in $\boldsymbol{e}$; spindle correlation: 0.24 in $\boldsymbol{d}$ and 0.89 in $\boldsymbol{e}$; see red dashed lines). $\boldsymbol{B}$, Recorded positions of the LFP in A (Figure legend continues.) 

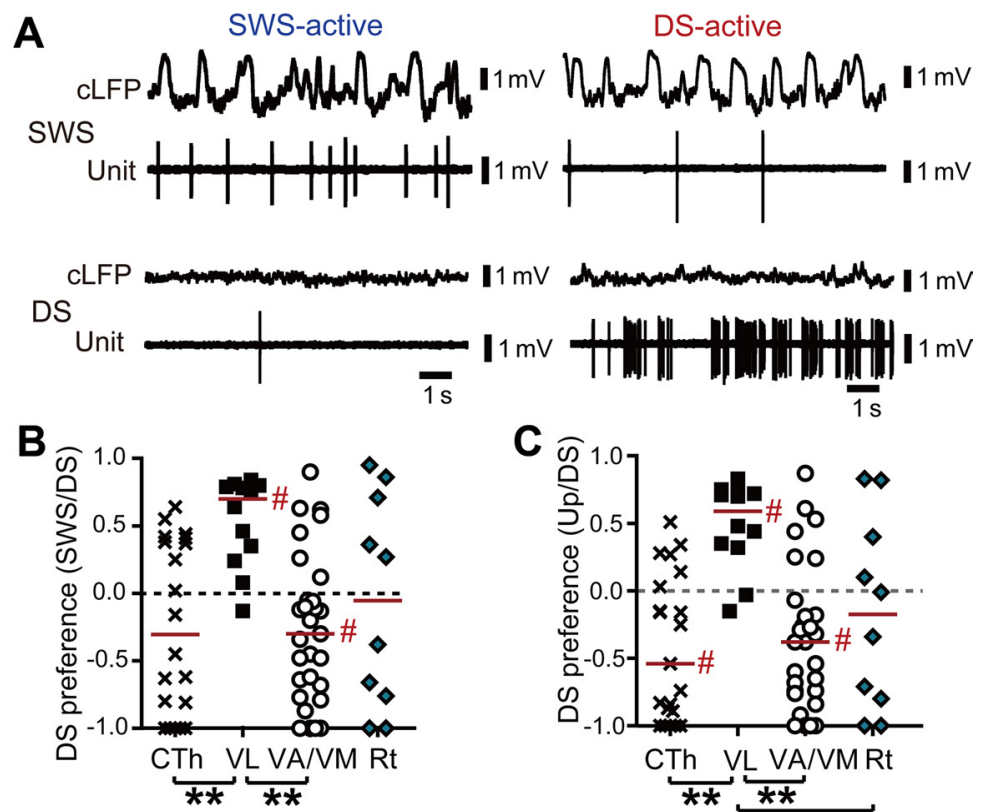

D

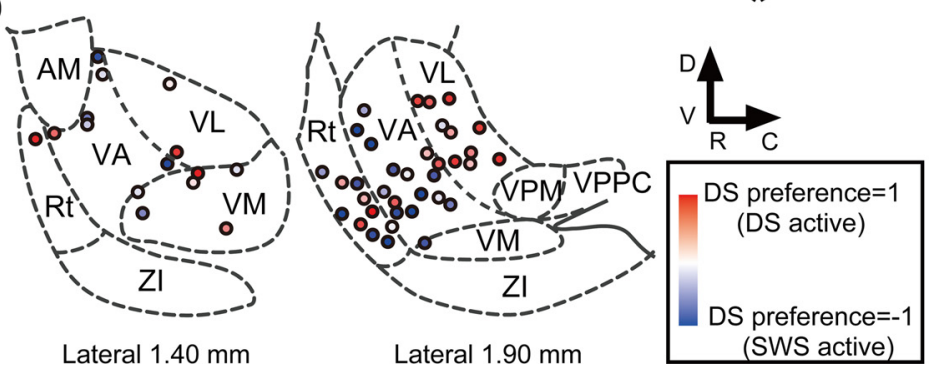

Figure 12. State dependency of firing. A, Firing frequency changes from SWS (top) to DS (bottom). In SWS, the LFP showed clear Up/Down rhythms, whereas the LFP was much lower in amplitude and more uniform in the DS. Some cells decreased their firing frequency from SWS to DS (left, SWS-active), while other cells increased activity in DS (right, DS-active). cLFP, Cortical LFP recorded in layer L5. B, DS preference of firing compared with SWS: (DSf - SWSf)/(DSf + SWSf), where DSf is the firing frequency in DS and SWSf in SWS. DS preference was different among four subtypes ( $p<0.01$, ANOVA; ${ }^{* *} p<0.01$, CTh vs VL and VA/VM vs VL). Those of VA/VM cells were skewed to negative values ( ${ }^{\#} p<0.05$ ), while those of VL cells to positive values ( ${ }^{\#} p<0.01$ ). CTh and Rt cells were diverse in DS preference. C, DS preference of firing compared with Up states: (DSf - Upf)/(DSf + Upf), where Upf is the firing frequency in slow-wave Up states. DS-state preference was different among four subtypes ( $p<0.01$, ANOVA; ${ }^{* *} p<0.01$, CTh vs VL and VA/VM vs VL; ${ }^{*} p<0.05$, VL vs Rt). Those of (Th and VA/VM cells were skewed to negative values, while those of VL cells to positive values ( ${ }^{\#} p<0.01$ ). Rt cells were diverse in DS preference. $D$, Spatial distribution of recorded cells with individual DS preferences compared with SWS in the thalamus (two thalamic sagittal planes, 1.4 and $1.9 \mathrm{~mm}$ lateral). DS preference from SWS to DS was expressed using a color code scale from red (1) to blue $(-1)$. SWS-active cells were found more in VA/VM, whereas DS-active cells were located more in VL. D, Dorsal; $V$, ventral; $R$, rostral; $C$, caudal.

Temporal specificity in Up-state firing

The functional role of Up depolarization in slow-wave sleep is not well understood. Since artificially enhanced slow-wave oscillations during sleep promote memory consolidation (Marshall et

\section{$\leftarrow$}

(Figure legend continued.) with the VGLUT2 immunoreaction (green fluorescence). Recorded positions of LFP at $1000 \mu \mathrm{m}$ depth and reference (ref.) were marked with Neurobiotin (yellow). C, Waveform correlation and root-mean-square (RMS) amplitude ratio of slow wave and spindles between a given depth and the deepest reference. Slow-wave RMS amplitude ratio decreased transiently at the middle ( 500 to $1000 \mu \mathrm{m}$ deep: from L2/3 to L5), and waveform correlation was inverted from negative to positive values there. Similarly, spindle RMS amplitude ratio decreased transiently at the middle, and waveform correlation was inverted around $1000 \mu \mathrm{m}$ deep (L5). D, Coincidence of spindle peaks/troughs between two depths. $\boldsymbol{a}$, Temporal correlation between spindle peaks in the superficial and troughs in the deepest reference $(\boldsymbol{a}$ in $\boldsymbol{A}) \cdot \boldsymbol{b}$, Correlation between spindle troughs in the deeper layer and troughs in the deepest reference $(\boldsymbol{e}$ in $\boldsymbol{A})$. Both histograms showed prominent peak around time lag $=0$, indicating spindle coincidence between layers. Neighboring peaks in the event correlogram located around $\sim 0.1 \mathrm{~s}$, representing synchronized spindles $(\sim 10 \mathrm{~Hz})$. al., 2006), it is speculated that reorganization of cortical neural circuits occurs during the Up states of SWS. Indeed, it is reported that firing patterns in hippocampal and cortical neurons during sleep reflect those during the awake state (Louie and Wilson, 2001; Euston et al., 2007). To organize temporal patterns of neural firing precisely, well coordinated and neuron subtype-specific mechanisms may be essential. In frontal cortex, some cortical fast spiking (FS) cells fire at specific temporal phases in Up states in SWS (Puig et al., 2008). In somatosensory thalamus, cell type- and phasic-specific firing during SWS has been reported (Slézia et al., 2011). We observed that only a few CTh neurons exhibited specificity of firing times during Up states, while the activity of many thalamic cells, especially in VA/VM and Rt nuclei, was temporally correlated with specific phases of Up/Down oscillations, with some neurons firing preferentially in the first half of Up states. These results suggest different groups of thalamic cells play specific roles during Up states. We found many thalamic cells fire during the early Up state, suggesting they may play a role in Up-state initiation and maintenance. In contrast, it is possible that decreased thalamocortical excitatory input in the later part of Up states contributes to Up-state termination.

Phase-specific activity in both excitatory thalamocortical neurons and in inhibitory FS cells might be essential to organize precise firing sequences in cortex. A previous study reported that some cortical FS cells tend to fire in the first half of Up states (early-FS cells), while others fire in the second half (late-FS cells), and that action potential timing in both FS cell types is coupled to gamma oscillations (Puig et al., 2008). Together, these observations suggest the following scenario: on receiving thalamic input, early-FS cells may generate gamma oscillations during the earlier part of Up states, whereas late-FS cells may sustain gamma in later periods of Up states by responding to the recurrent excitation of pyramidal neurons during the SWS.

\section{Generation of synchronous Up states and maintenance of slow wave}

Up-state depolarization is thought to be generated in neocortex (Sanchez-Vives and McCormick, 2000; Timofeev et al., 2000; Sakata and Harris, 2009). Under in vivo conditions, however, some thalamic neurons discharge spikes earlier than Up-state initiation (Contreras and Steriade, 1995). In support of this, when connections between the thalamus and cortex are severed in thalamocortical slices, the incidence of spontaneous cortical Up states is reduced (Rigas and Castro-Alamancos, 2007). On the basis of these findings, Crunelli and Hughes (2010) proposed the possibility that the thalamus contributes to cortical Up states. Even though cortical Up states are mainly caused by recurrent 
Table 1. Firing frequency in each subtype

\begin{tabular}{|c|c|c|c|c|c|}
\hline & CTh & $\mathrm{VL}$ & VA/VM & Rt & ANOVA \\
\hline \multicolumn{6}{|l|}{ SWS firing frequency $(\mathrm{Hz})$} \\
\hline Whole & $\begin{array}{l}1.20 \pm 0.98 \\
{[1.06]}\end{array}$ & $\begin{array}{l}1.26 \pm 1.02^{\dagger+} \\
{[0.94]}\end{array}$ & $\begin{array}{l}2.82 \pm 4.32^{\dagger} \\
{[1.37]}\end{array}$ & $\begin{array}{l}1.89 \pm 1.69 \\
{[1.31]}\end{array}$ & $p>0.05$ \\
\hline Up state & $\begin{array}{l}2.06 \pm 1.32^{\dagger} \\
{[1.61]}\end{array}$ & $\begin{array}{l}1.37 \pm 0.74^{t \dagger} \\
{[1.33]}\end{array}$ & $\begin{array}{l}3.64 \pm 5.66^{t \dagger} \\
{[1.84]}\end{array}$ & $\begin{array}{l}2.86 \pm 2.36 \\
{[1.77]}\end{array}$ & $p>0.05$ \\
\hline Down state & $\begin{array}{l}0.28 \pm 0.28 \\
{[0.24]} \\
(n=25)\end{array}$ & $\begin{array}{l}0.77 \pm 0.56 \\
{[0.61]} \\
(n=12)\end{array}$ & $\begin{array}{l}1.37 \pm 2.82 \\
{[0.64]} \\
(n=30)\end{array}$ & $\begin{array}{l}0.92 \pm 1.17 \\
{[0.31]} \\
(n=10)\end{array}$ & $p>0.05$ \\
\hline DS firing frequency $(\mathrm{Hz})$ & $\begin{array}{l}1.70 \pm 2.78 \\
{[0.40]} \\
(n=21)\end{array}$ & $\begin{array}{l}5.40 \pm 4.75 \\
{[4.00]} \\
(n=12)\end{array}$ & $\begin{array}{l}2.06 \pm 2.82 \\
{[0.95]} \\
(n=29)\end{array}$ & $\begin{array}{l}4.94 \pm 6.75 \\
{[1.60]} \\
(n=10)\end{array}$ & $p<0.05$ \\
\hline
\end{tabular}

Shown are mean $\pm S D$ [median]; $n$, number of cells. SWS, Slow-wave state; Up state and Down state in the slow wave; DS, desynchronized state.

${ }^{\dagger} p<0.05 ;{ }^{\dagger \dagger} p<0.01$, Mann-Whitney test for SWS or Up state versus DS firing frequency.

Table 2. Up/Down time-, spindle phase-, and state-dependent firing different between $\mathrm{L} 5$ corticothalamic cells and thalamic subregions

\begin{tabular}{|c|c|c|c|c|}
\hline & \multirow{2}{*}{$\begin{array}{l}\text { Cortex } \\
\text { L5 CTh }\end{array}$} & \multicolumn{3}{|l|}{ Thalamus } \\
\hline & & VL & VA/VM & $\mathrm{Rt}$ \\
\hline Burst occurrence & Low & High & High & Low $\sim$ high \\
\hline \multicolumn{5}{|l|}{ Up/Down states } \\
\hline Down firing & $( \pm)$ & $(+)$ & $(+)$ & $(+)$ \\
\hline Down-to-Up firing cells & Few & Few & Some & Some \\
\hline Temporal specificity within Up & $(+)$ & $(+)$ & $(++)$ & $(++)$ \\
\hline \multicolumn{5}{|l|}{ Spindles } \\
\hline Modulation strength & $(+)$ & $(+)$ & $(+)$ & $(++)$ \\
\hline Firing phase (L5 LFP) & Trough & Peak & Peak & Peak \\
\hline \multicolumn{5}{|l|}{ State dependency } \\
\hline SWS versus DS & & DS-active & SWS-active & \\
\hline Up states versus DS & Up-active & DS-active & Up-active & \\
\hline
\end{tabular}

excitation among pyramidal cells, the balance of excitation and inhibition is essential to maintain a well organized rhythm (Shu et al., 2003). Thalamocortical excitation may also be involved in maintaining this balance.

Our data demonstrate selective increases in firing frequency near Up-state initiations in some VA/VM and Rt cells. Neurons in VA/VM extend their axons to L1 of frontal cortex (Kuramoto et al., 2009; Rubio-Garrido et al., 2009). L5 CTh cells have a dense tuft structure in L1 (Hirai et al., 2012). Thus, it is proposed that some populations of VA/VM and Rt cells are excited in advance of Up-state initiation, by which they may help initiate the cortical Up state. Excited L5 CTh cells, in turn, synchronize the thalamic nuclei in slow oscillations. At the same time, some Rt neurons start to fire in spindle rhythms to recruit thalamic relay cells that transmit the synchronous spindle activity to the cortex. In response to spindle input from the thalamus, L5 pyramidal cells may discharge with some delay, producing firing time lags between thalamic and cortical cells. L5 CTh cells, however, would not affect spindle generation or the firing time specificity during Up states in Rt because they do not project to the reticular nucleus (Ojima, 1994; Jones, 2007).

The thalamic VM nucleus and reticular part of substantia nigra (SNR) are implicated in the manifestation of absence seizures, which are characterized by a sudden loss of consciousness with EEG changes (Danober et al., 1998; Paz et al., 2007). Appropriate timing and strength of inhibitory inputs from the Rt and SNR are supposed to be important for normal Up/Down oscillations and stable consciousness supported by cortical desynchronization.

In M2, L2/3a pyramidal cells project to the amygdala or parahippocampal cortex (Gabbott et al., 2005; Hirai et al., 2012), and
L5 CTh cells also innervate the striatum (Lévesque and Parent, 1998), revealing the close relationship of M2 with the declarative memory-related areas. Like other cortical areas, frontal cortical L2/3 pyramidal cells make feedforward excitatory connections with L5 pyramidal cells (Otsuka and Kawaguchi, 2008). During SWS, pyramidal cells in L2/3a were more active than those in $\mathrm{L} 2 / 3 \mathrm{~b}$, the middle thalamic input-recipient zone in M2. Similarly, in rodent barrel cortex, sensory responses are suppressed during Up states in the middle thalamic input-recipient zone (Petersen et al., 2003; Sachdev et al., 2004; Castro-Alamancos, 2009). Thus, the pyramidal subnetworks in L2/3a and L5 in frontal cortex were specifically activated during SWS in cooperation with the VA/VM system, further suggesting importance of slow wave in declarative memory consolidation.

\section{Spindle modulation during Up states}

Spindle waves are generated in the thalamus and transmitted to the cortex (Steriade et al., 1987; Contreras and Steriade, 1996). Spike timing during spindle cycles was different between corticothalamic and thalamocortical cells. Thalamus-induced excitatory currents in upper L1 and L2/3b (corresponding to L4 in other cortical areas) may summate over several tens of milliseconds to induce delayed activity in L5 pyramidal neurons. Another explanation for the delay between thalamic input and action potential generation in L5 neurons during the spindle is that thalamocortical inputs may initially activate cortical GABAergic neurons that then transiently inhibit pyramidal cells to produce the phase lag.

Excitatory inputs occurring during the spindle rhythm facilitate long-term potentiation in pyramidal cells (Rosanova and Ulrich, 2005). The difference in spike timing during a spindle cycle in thalamic neurons and cortical pyramidal cells may support independent regulation of synaptic plasticity for cortical recurrent and thalamic inputs onto L5 pyramidal cells. Because L5 CTh cells do not project to Rt nucleus, they will not interfere with rhythm generation in Rt nucleus. This may help sustain the firing time lag between thalamic and cortical cells.

\section{Brain state dependency of firing frequency}

The firing frequency change observed from SWS to DS was different among neuron subtypes. The cerebellar nuclei send excitatory inputs to VL, whereas the basal ganglia send inhibitory inputs to VA/VM areas (Asanuma et al., 1983; Ilinsky and KultasIlinsky, 1987; Sakai et al., 1996). These data raise the possibility that increased spontaneous activity in the cerebellum and basal ganglia during DS enhances the spontaneous activity of VL cells while suppressing activity in VA/VM area. Activity enhancement 
in sensory thalamic nuclei may induce sensory area desynchronization (Hirata and Castro-Alamancos, 2010). The frontal cortex would be desynchronized by increases in the output of cerebellum-linked thalamic nuclei.

As described above, we observed enhanced activity of VA/VM cells during the initiation of Up states that may contribute to Up-state initiation. To evoke Up states, spontaneous activity during Down states may be necessary, which may be regulated by inhibitory input from the substantia nigra. In the awake condition, inhibitory input to VA/VM nuclei would become enhanced and this unbalance of excitation and inhibition may contribute to the cessation of the slow oscillation. The strong relationship of neural firing in basal ganglia-linked thalamic nuclei with Upstate initiation and spindle activity indicates that the basal ganglia may indirectly set the activation level of neocortex via control of thalamocortical drive. These results suggest the crucial role of loop circuits between the frontal cortex, basal ganglia, and thalamus not only in motor regulation during awake states but also during sleep oscillations and during the transition between sleep and wakefulness.

\section{References}

Amzica F, Steriade M (1995) Short- and long-range neuronal synchronization of the slow $(<1 \mathrm{~Hz})$ cortical oscillation. J Neurophysiol 73:20-38.

Arai R, Jacobowitz DM, Deura S (1994) Distribution of calretinin, calbindin-D28k, and parvalbumin in the rat thalamus. Brain Res Bull 33:595-614.

Asanuma C, Thach WT, Jones EG (1983) Distribution of cerebellar terminations and their relation to other afferent terminations in the ventral lateral thalamic region of the monkey. Brain Res 286:237-265.

Buzsáki G (1998) Memory consolidation during sleep: a neurophysiological perspective. J Sleep Res 7 [Suppl 1]:17-23.

Castro-Alamancos MA (2009) Cortical up and activated states: implications for sensory information processing. Neuroscientist 15:625-634.

Contreras D, Steriade M (1995) Cellular basis of EEG slow rhythms: a study of dynamic corticothalamic relationships. J Neurosci 15:604-622.

Contreras D, Steriade M (1996) Spindle oscillation in cats: the role of corticothalamic feedback in a thalamically generated rhythm. J Physiol 490:159-179.

Cowan RL, Wilson CJ (1994) Spontaneous firing patterns and axonal projections of single corticostriatal neurons in the rat medial agranular cortex. J Neurophysiol 71:17-32.

Crunelli V, Hughes SW (2010) The slow $(<1 \mathrm{~Hz})$ rhythm of non-REM sleep: a dialogue between three cardinal oscillators. Nat Neurosci 13:9-17.

Danober L, Deransart C, Depaulis A, Vergnes M, Marescaux C (1998) Pathophysiological mechanisms of genetic absence epilepsy in the rat. Prog Neurobiol 55:27-57.

Deschênes M, Paradis M, Roy JP, Steriade M (1984) Electrophysiology of neurons of lateral thalamic nuclei in cat: resting properties and burst discharges. J Neurophysiol 51:1196-1219.

Destexhe A, Hughes SW, Rudolph M, Crunelli V (2007) Are corticothalamic "up" states fragments of wakefulness? Trends Neurosci 30:334-342.

Euston DR, Tatsuno M, McNaughton BL (2007) Fast-forward playback of recent memory sequences in prefrontal cortex during sleep. Science 318:1147-1150.

Gabbott PL, Warner TA, Jays PR, Salway P, Busby SJ (2005) Prefrontal cortex in the rat: projections to subcortical autonomic, motor, and limbic centers. J Comp Neurol 492:145-177.

Hirai Y, Morishima M, Karube F, Kawaguchi Y (2012) Specialized cortical subnetworks differentially connect frontal cortex to parahippocampal areas. J Neurosci, in press.

Hirata A, Castro-Alamancos MA (2010) Neocortex network activation and deactivation states controlled by the thalamus. J Neurophysiol 103:1147-1157.

Ilinsky IA, Kultas-Ilinsky K (1987) Sagittal cytoarchitectonic maps of the Macaca mulatta thalamus with a revised nomenclature of the motorrelated nuclei validated by observations on their connectivity. J Comp Neurol 262:331-364
Jones EG (2001) The thalamic matrix and thalamocortical synchrony. Trends Neurosci 24:595-601.

Jones EG (2007) The thalamus, 2nd edition. Cambridge, UK: Cambridge UP.

Kuramoto E, Furuta T, Nakamura KC, Unzai T, Hioki H, Kaneko T (2009) Two types of thalamocortical projections from the motor thalamic nuclei of the rat: a single neuron-tracing study using viral vectors. Cereb Cortex 19:2065-2077.

Lévesque M, Parent A (1998) Axonal arborization of corticostriatal and corticothalamic fibers arising from prelimbic cortex in the rat. Cereb Cortex 8:602-613

Louie K, Wilson MA (2001) Temporally structured replay of awake hippocampal ensemble activity during rapid eye movement sleep. Neuron 29:145-156.

Marshall L, Helgadóttir H, Mölle M, Born J (2006) Boosting slow oscillations during sleep potentiates memory. Nature 444:610-613.

Metherate R, Ashe JH (1993) Ionic flux contributions to neocortical slow waves and nucleus basalis-mediated activation: whole-cell recordings in vivo. J Neurosci 13:5312-5323.

Morishima M, Kawaguchi Y (2006) Recurrent connection patterns of corticostriatal pyramidal cells in frontal cortex. J Neurosci 26:4394-4405.

Morishima M, Morita K, Kubota Y, Kawaguchi Y (2011) Highly differentiated projection-specific cortical subnetworks. J Neurosci 31:10380-10391.

Mukovski M, Chauvette S, Timofeev I, Volgushev M (2007) Detection of active and silent states in neocortical neurons from the field potential signal during slow-wave sleep. Cereb Cortex 17:400-414.

Nowak LG, Azouz R, Sanchez-Vives MV, Gray CM, McCormick DA (2003) Electrophysiological classes of cat primary visual cortical neurons in vivo as revealed by quantitative analyses. J Neurophysiol 89:1541-1566.

Ojima H (1994) Terminal morphology and distribution of corticothalamic fibers originating from layers 5 and 6 of cat primary auditory cortex. Cereb Cortex 4:646-663.

Otsuka T, Kawaguchi Y (2008) Firing-pattern-dependent specificity of cortical excitatory feedforward subnetworks. J Neurosci 28:11186-11195.

Otsuka T, Kawaguchi Y (2011) Cell diversity and connection specificity between callosal projection neurons in the frontal cortex. J Neurosci 31:3862-3870.

Paz JT, Chavez M, Saillet S, Deniau JM, Charpier S (2007) Activity of ventral medial thalamic neurons during absence seizures and modulation of cortical paroxysms by the nigrothalamic pathway. J Neurosci 27:929-941.

Petersen CC, Hahn TT, Mehta M, Grinvald A, Sakmann B (2003) Interaction of sensory responses with spontaneous depolarization in layer $2 / 3$ barrel cortex. Proc Natl Acad Sci U S A 100:13638-13643.

Pinault D (1996) A novel single-cell staining procedure performed in vivo under electrophysiological control: morpho-functional features of juxtacellularly labeled thalamic cells and other central neurons with biocytin or Neurobiotin. J Neurosci Methods 65:113-136.

Pinault D (2004) The thalamic reticular nucleus: structure, function and concept. Brain Res Brain Res Rev 46:1-31.

Puig MV, Ushimaru M, Kawaguchi Y (2008) Two distinct activity patterns of fast-spiking interneurons during neocortical UP states. Proc Natl Acad Sci U S A 105:8428-8433.

Rigas P, Castro-Alamancos MA (2007) Thalamocortical Up states: differential effects of intrinsic and extrinsic cortical inputs on persistent activity. J Neurosci 27:4261-4272.

Rosanova M, Ulrich D (2005) Pattern-specific associative long-term potentiation induced by a sleep spindle-related spike train. J Neurosci 25:9398-9405.

Rubio-Garrido P, Pérez-de-Manzo F, Porrero C, Galazo MJ, Clascá F (2009) Thalamic input to distal apical dendrites in neocortical layer 1 is massive and highly convergent. Cereb Cortex 19:2380-2395.

Sachdev RN, Ebner FF, Wilson CJ (2004) Effect of subthreshold up and down states on the whisker-evoked response in somatosensory cortex. J Neurophysiol 92:3511-3521.

Sakai ST, Inase M, Tanji J (1996) Comparison of cerebellothalamic and pallidothalamic projections in the monkey (Macaca fuscata): a double anterograde labeling study. J Comp Neurol 368:215-228.

Sakata S, Harris KD (2009) Laminar structure of spontaneous and sensoryevoked population activity in auditory cortex. Neuron 64:404-418.

Sanchez-Vives MV, McCormick DA (2000) Cellular and network mech- 
anisms of rhythmic recurrent activity in neocortex. Nat Neurosci 3:1027-1034.

Sherman SM, Guillery RW (1998) On the actions that one nerve cell can have on another: distinguishing "drivers" from "modulators." Proc Natl Acad Sci U S A 95:7121-7126.

Shu Y, Hasenstaub A, McCormick DA (2003) Turning on and off recurrent balanced cortical activity. Nature 423:288-293.

Slézia A, Hangya B, Ulbert I, Acsády L (2011) Phase advancement and nucleus-specific timing of thalamocortical activity during slow cortical oscillation. J Neurosci 31:607-617.

Steriade M (2006) Grouping of brain rhythms in corticothalamic systems. Neuroscience 137:1087-1106.

Steriade M, Domich L, Oakson G, Deschênes M (1987) The deafferented reticular thalamic nucleus generates spindle rhythmicity. J Neurophysiol 57:260-273.
Steriade M, Nuñez A, Amzica F (1993) A novel slow ( $<1 \mathrm{~Hz}$ ) oscillation of neocortical neurons in vivo: depolarizing and hyperpolarizing components. J Neurosci 13:3252-3265.

Steriade M, Timofeev I, Grenier F (2001) Natural waking and sleep states: a view from inside neocortical neurons. J Neurophysiol 85:1969-1985.

Tierney PL, Dégenètais E, Thierry AM, Glowinski J, Gioanni Y (2004) Influence of the hippocampus on interneurons of the rat prefrontal cortex. Eur J Neurosci 20:514-524.

Timofeev I, Grenier F, Bazhenov M, Sejnowski TJ, Steriade M (2000) Origin of slow cortical oscillations in deafferented cortical slabs. Cereb Cortex 10:1185-1199.

Volgushev M, Chauvette S, Mukovski M, Timofeev I (2006) Precise longrange synchronization of activity and silence in neocortical neurons during slow-wave oscillations [corrected]. J Neurosci 26:5665-5672. 